\title{
On the third secant variety
}

\author{
Jarosław Buczyński • J.M. Landsberg
}

Received: 12 January 2012 / Accepted: 4 September 2012 / Published online: 16 January 2014

(C) The Author(s) 2014. This article is published with open access at Springerlink.com

\begin{abstract}
We determine normal forms and ranks of tensors of border rank at most three. We present a differential-geometric analysis of limits of secant planes in a more general context. In particular there are at most four types of points on limiting trisecant planes for cominuscule varieties such as Grassmannians. We also show that the singular locus of the secant varieties $\sigma_{r}\left(\operatorname{Seg}\left(\mathbb{P}^{n} \times \mathbb{P}^{m} \times \mathbb{P}^{q}\right)\right)$ has codimension at least two for $r=2,3$.
\end{abstract}

Keywords Secant varieties · Border rank - Tensor rank · Segre product ·

Cominuscule varieties $\cdot$ Singularities of secant varieties $\cdot$ Classification of points of border rank three

\section{Introduction}

Throughout the paper we work over the field of complex numbers $\mathbb{C}$.

Motivated by applications, there has been a considerable amount of recent research on ranks and border ranks of tensors, see, e.g., [9, 15] and references therein. In signal processing one is interested in determining ranks of tensors, see, e.g., [6] and

J. Buczyński

Institut Fourier, Université Grenoble I, 100 rue des Maths, BP 74, 38402 St Martin d'Hères cedex, France

J. Buczyński ( $\square)$

Institute of Mathematics of the Polish Academy of Sciences, ul. Śniadeckich 8, P.O. Box 21, 00-956

Warszawa, Poland

e-mail: jabu@mimuw.edu.pl

J.M. Landsberg

Department of Mathematics, Texas A\&M University, Mailstop 3368, College Station, TX

77843-3368, USA

e-mail: jml@math.tamu.edu 
references therein. In computational complexity, one looks for exotic algorithms via limits of tensors of a given rank, see [16]. There are adequate tests to determine the border ranks of tensors of small border rank; however, the possible ranks of such tensors are not well understood. In this article we present normal forms for tensors of border rank three. Already in this case the problem becomes subtle. We work in the more general setting of secant varieties.

\subsection{Definitions, notational conventions}

For a projective variety $X \subset \mathbb{P} V$ not contained in a hyperplane, the $X$-rank of $p \in$ $\mathbb{P} V, R_{X}(p)$, is defined to be the smallest $r$ such that there exist $x_{1}, \ldots, x_{r} \in X$ such that $p$ is in the span of $x_{1}, \ldots, x_{r}$, and the $X$-border rank $\underline{\mathbf{R}}_{X}(p)$ is defined to be the smallest $r$ such that there exist curves $x_{1}(t), \ldots, x_{r}(t) \in X$ such that $p$ is in the span of the limiting plane $\lim _{t \rightarrow 0}\left\langle x_{1}(t), \ldots, x_{r}(t)\right\rangle$. Let $\sigma_{r}(X) \subset \mathbb{P} V$ denote the set of points of $X$-border rank at most $r$. When $X=\operatorname{Seg}\left(\mathbb{P} A_{1} \times \cdots \times \mathbb{P} A_{n}\right) \subset \mathbb{P}\left(A_{1} \otimes\right.$ $\left.\cdots \otimes A_{n}\right)$ is the set of rank-one tensors in a space of tensors, the $X$-rank and border rank agree with the usual notions of tensor rank and border rank. The set of points of $X$-rank $r$ contains a Zariski open subset of $\sigma_{r}(X)$ and we are interested in the complement of this set.

We let $\sigma_{r}^{0}(X)$ denote the points of $\sigma_{r}(X)$ of rank $r$. The tangential variety of a smooth variety $X \subset \mathbb{P} V, \tau(X) \subset \mathbb{P} V$, consists of all points on all embedded tangent $\mathbb{P}^{1}$ 's. For varieties $X, Y \subset \mathbb{P} V$, define

$$
J(X, Y):=\overline{\{p \in \mathbb{P} V \mid \exists x \in X, y \in Y \text { such that } p \in\langle x, y\rangle\}},
$$

the join of $X$ and $Y$. Note that $J(X, X)=\sigma_{2}(X)$. For a set $Z \subset \mathbb{P} V, \hat{Z} \subset V$ denotes the cone over it and $\langle Z\rangle$ its linear span. For a variety $Y \subset \mathbb{P} V, Y_{\text {sing }}$ denotes the singular points of $Y$. The affine tangent space to a variety $X \subset \mathbb{P} V$ at a smooth point $x$ is denoted $\hat{T}_{x} X \subset V$.

Throughout the paper we assume $A_{1}, \ldots, A_{n}, A, B, C$ to be complex vector spaces of dimension at least 2 .

\subsection{Results on ranks and normal forms for tensors}

The following proposition was probably "known to the experts" but we did not find it in the literature, so we include a statement and proof.

Proposition 1.1 Let $X=\operatorname{Seg}\left(\mathbb{P} A_{1} \times \cdots \times \mathbb{P} A_{n}\right) \subset \mathbb{P}\left(A_{1} \otimes \cdots \otimes A_{n}\right)$ be a Segre variety. There is a normal form for points $x \in \hat{\sigma}_{2}(X)$ :

(a) $x=a_{1}^{1} \otimes \cdots \otimes a_{1}^{n}$ for a point of $X$, which has rank 1 ;

(b) $x=a_{1}^{1} \otimes \cdots \otimes a_{1}^{n}+a_{2}^{1} \otimes \cdots \otimes a_{2}^{n}$ for a point on a secant line to $X$ (here we require at least two of the $a_{2}^{i}$ 's to be independent of the corresponding $a_{1}^{i}$ ), which has rank 2; and

(c) for each $J \subseteq\{1, \ldots, n\},|J|>2$, the normal form

$$
x=\sum_{j \in J} a_{1}^{1} \otimes \cdots \otimes a_{1}^{j-1} \otimes a_{2}^{j} \otimes a_{1}^{j+1} \otimes \cdots \otimes a_{1}^{n}
$$


where each $a_{2}^{j}$ is independent of the corresponding $a_{1}^{j}$. This case has rank $|J|$.

In particular, all ranks from 1 to $n$ occur for elements of $\sigma_{2}(X)$.

Our main result is the analogous classification for points in the third secant variety of the Segre product:

Theorem 1.2 Assume $n \geq 3$ and let $X:=\operatorname{Seg}\left(\mathbb{P} A_{1} \times \cdots \times \mathbb{P} A_{n}\right)$. Let $p=[v] \in$ $\sigma_{3}(X) \backslash \sigma_{2}(X)$. Then $v$ has one of the following normal forms:

(i) $v=x+y+z$ with $[x],[y],[z] \in X$;

(ii) $v=x^{\prime}+y$, with $[x],[y] \in X$ and $x^{\prime} \in \hat{T}_{[x]} X$;

(iii) $v=x^{\prime}+x^{\prime \prime}$, where $[x(t)] \subset X$ is a curve and $x^{\prime}=x^{\prime}(0), x^{\prime \prime}=x^{\prime \prime}(0)$; or

(iv) $v=x^{\prime}+y^{\prime}$, where $[x],[y] \in X$ are distinct points that lie on a line contained in $X, x^{\prime} \in \hat{T}_{[x]} X$, and $y^{\prime} \in \hat{T}_{[y]} X$.

The points of type (i) contain a Zariski open subset of $\sigma_{3}(X) \backslash \sigma_{2}(X)$. If $\operatorname{dim} A_{i} \geq 3$, then those of type (ii) have codimension one in $\sigma_{3}(X)$, those of type (iii) are contained in the closure of those of type (ii) and have codimension two in $\sigma_{3}(X)$, and those of type (iv) are in the closure of the set of points of type (iii) and have codimension four in $\sigma_{3}(X)$. There are $n$ distinct components of points of type (iv). A general point of each type is not a point of any of the other types.

When $n=2$, all points on $\sigma_{3}\left(\operatorname{Seg}\left(\mathbb{P} A_{1} \times \mathbb{P} A_{2}\right)\right) \backslash \sigma_{2}\left(\operatorname{Seg}\left(\mathbb{P} A_{1} \times \mathbb{P} A_{2}\right)\right)$ are of type (i).

The following result may also have been "known to the experts" but we did not find it in the literature either.

Theorem 1.3 A general point of $\tau(\operatorname{Seg}(\mathbb{P} A \times \mathbb{P} B \times \mathbb{P} C))$, i.e., a point with the normal form (1.1) with $|J|=3$, is a smooth point of $\sigma_{2}(\operatorname{Seg}(\mathbb{P} A \times \mathbb{P} B \times \mathbb{P} C))$. In particular,

$$
\operatorname{codim}\left(\sigma_{2}(\operatorname{Seg}(\mathbb{P} A \times \mathbb{P} B \times \mathbb{P} C))_{\text {sing }}, \sigma_{2}(\operatorname{Seg}(\mathbb{P} A \times \mathbb{P} B \times \mathbb{P} C))\right) \geq 2
$$

We prove an analogous result for $\sigma_{3}(\operatorname{Seg}(\mathbb{P} A \times \mathbb{P} B \times \mathbb{P} C))$ :

Theorem 1.4 Let $p \in \sigma_{3}(\operatorname{Seg}(\mathbb{P} A \times \mathbb{P} B \times \mathbb{P} C))$. If $p$ is a general point of type (ii) or (iii), or a general point of any component of points of type (iv), then $p$ is a nonsingular point of $\sigma_{3}(\operatorname{Seg}(\mathbb{P} A \times \mathbb{P} B \times \mathbb{P} C))$. Moreover, if $\operatorname{dim} A, \operatorname{dim} B, \operatorname{dim} C \geq 3$, and $p$ is a general point in the set of the points contained in some $\mathbb{P}\left(\mathbb{C}^{2} \otimes \mathbb{C}^{3} \otimes \bar{C}^{3}\right)$, then $p$ is a non-singular point of $\sigma_{3}(\operatorname{Seg}(\mathbb{P} A \times \mathbb{P} B \times \mathbb{P} C))$, and similarly for permuted statements.

In particular, $\operatorname{codim}\left(\sigma_{3}(\operatorname{Seg}(\mathbb{P} A \times \mathbb{P} B \times \mathbb{P} C))_{\text {sing }}, \sigma_{3}(\operatorname{Seg}(\mathbb{P} A \times \mathbb{P} B \times \mathbb{P} C))\right) \geq 2$.

Normal forms for Theorem 1.2 when $n=3$ are the following:

(i) $a_{1} \otimes b_{1} \otimes c_{1}+a_{2} \otimes b_{2} \otimes c_{2}+a_{3} \otimes b_{3} \otimes c_{3}$,

(ii) $a_{1} \otimes b_{1} \otimes c_{2}+a_{1} \otimes b_{2} \otimes c_{1}+a_{2} \otimes b_{1} \otimes c_{1}+a_{3} \otimes b_{3} \otimes c_{3}$,

(iii) $a_{1} \otimes b_{2} \otimes c_{2}+a_{2} \otimes b_{1} \otimes c_{2}+a_{2} \otimes b_{2} \otimes c_{1}+a_{1} \otimes b_{1} \otimes c_{3}+a_{1} \otimes b_{3} \otimes c_{1}+a_{3} \otimes b_{1} \otimes c_{1}$, 
(iv) $a_{2} \otimes b_{1} \otimes c_{2}+a_{2} \otimes b_{2} \otimes c_{1}+a_{1} \otimes b_{1} \otimes c_{3}+a_{1} \otimes b_{3} \otimes c_{1}+a_{3} \otimes b_{1} \otimes c_{1}$.

For type (iv) there are two other normal forms, where the role of $a$ is switched with that of $b$ and $c$. These normal forms are depicted in terms of "slices" in Table 1. (In the tensor literature, 3-way tensors $T \in A \otimes B \otimes C$ are often studied by their images $T\left(A^{*}\right) \subset B \otimes C$, etc., and these images are studied in terms of bases, resulting in a parameterized subspace of a space of matrices. These parameterized spaces of matrices are called slices.) Here $a_{j}, b_{j}, c_{j}$ need not be independent vectors, so to parameterize the spaces, fix bases of each space and write the $a_{j}, b_{j}, c_{j}$ as arbitrary linear combinations of basis vectors. (However, there are some independence requirements.)

Here are normal forms for all $n$ :

$$
\begin{aligned}
p_{(\mathrm{i})}= & a_{1}^{1} \otimes \cdots \otimes a_{1}^{n}+a_{2}^{1} \otimes \cdots \otimes a_{2}^{n}+a_{3}^{1} \otimes \cdots \otimes a_{3}^{n} \\
p_{(\mathrm{ii})}= & \sum_{i} a_{1}^{1} \otimes \cdots \otimes a_{1}^{i-1} \otimes a_{2}^{i} \otimes a_{1}^{i+1} \otimes \cdots \otimes a_{1}^{n}+a_{3}^{1} \otimes \cdots \otimes a_{3}^{n} \\
p_{(\mathrm{iii})}= & \sum_{i<j} a_{1}^{1} \otimes \cdots \otimes a_{1}^{i-1} \otimes a_{2}^{i} \otimes a_{1}^{i+1} \otimes \cdots \otimes a_{1}^{j-1} \otimes a_{2}^{j} \otimes a_{1}^{j+1} \otimes \cdots \otimes a_{1}^{n} \\
& +\sum_{i} a_{1}^{1} \otimes \cdots \otimes a_{1}^{i-1} \otimes a_{3}^{i} \otimes a_{1}^{i+1} \otimes \cdots \otimes a_{1}^{n} \\
p_{(\mathrm{iv})}= & \sum_{s=2}^{n} a_{2}^{1} \otimes a_{1}^{2} \otimes \cdots \otimes a_{1}^{s-1} \otimes a_{2}^{s} \otimes a_{1}^{s+1} \otimes \cdots \otimes a_{1}^{n} . \\
& +\sum_{i=1}^{n} a_{1}^{1} \otimes \cdots \otimes a_{1}^{i-1} \otimes a_{3}^{i} \otimes a_{1}^{i+1} \otimes \cdots \otimes a_{1}^{n} .
\end{aligned}
$$

Again, (1.5) has $n-1$ other normal forms, where the role of $a_{*}^{1}$ is exchanged with $a_{*}^{i}$. Also, the vectors need not all be linearly independent.

Remark 1.5 In contrast to case (iv) above, already with four points on a three-factor Segre spanning a three-dimensional vector space, one can obtain new limits by taking a second derivative, even when the limiting points are distinct. Consider the points $x_{1}=a_{1} \otimes b_{1} \otimes c_{1}, x_{2}=a_{2} \otimes b_{2} \otimes c_{1}, x_{3}=\frac{1}{2}\left(a_{1}+a_{2}\right) \otimes\left(b_{1}-b_{2}\right) \otimes c_{1}, x_{4}=\frac{1}{2}\left(a_{1}-\right.$ $\left.a_{2}\right) \otimes\left(b_{1}+b_{2}\right) \otimes c_{1}$. Note that $x_{1}=x_{2}+x_{3}+x_{4}$. Here both first and second derivatives of curves give new points. More generally, consider

$$
\operatorname{Seg}(v_{2}\left(\mathbb{P}^{1}\right) \times \underbrace{\mathbb{P}^{0} \times \cdots \times \mathbb{P}^{0}}_{(n-2) \text { factors }}) \subset \operatorname{Seg}\left(\mathbb{P} A_{1} \otimes \cdots \otimes \mathbb{P} A_{n}\right) .
$$

Any four points lying on $\operatorname{Seg}\left(v_{2}\left(\mathbb{P}^{1}\right) \times \mathbb{P}^{0} \times \cdots \times \mathbb{P}^{0}\right)$ will be linearly dependent. Exceptional limit points turn out to be important-an exceptional limit in $\sigma_{5}(\operatorname{Seg}(\mathbb{P} A \times \mathbb{P} B \times \mathbb{P} C))$ is used in Bini's approximate algorithm to multiply $2 \times 2$ matrices with an entry zero, and an exceptional limit in $\sigma_{7}(\operatorname{Seg}(\mathbb{P} A \times \mathbb{P} B \times \mathbb{P} C))$ is used in Schönhage's approximate algorithm to multiply $3 \times 3$ matrices using 21 multiplications, see [3, Thm 1.15]. 
Since there are only finitely many configurations of triples of points in $A_{i}$ up to the action of $G L\left(A_{i}\right)$, we conclude:

Corollary 1.6 There are only finitely many orbits of the action of $G L\left(A_{1}\right) \times \cdots \times$ $G L\left(A_{n}\right)$ on $\sigma_{3}\left(\operatorname{Seg}\left(\mathbb{P} A_{1} \times \cdots \times \mathbb{P} A_{n}\right)\right)$.

In the three-factor case, there are 39 orbits, see Sect. 6.

Remark 1.7 Points of the form $y+y^{\prime}+y^{\prime \prime}$ where $y(t)$ is a curve on $\hat{\operatorname{Seg}}\left(\mathbb{P} A_{1} \otimes \cdots \otimes\right.$ $\left.\mathbb{P} A_{n}\right)$ have rank at most $\left(\begin{array}{c}n+1 \\ 2\end{array}\right)$ because all such points are of the form (1.4) (perhaps with linearly dependent variables). The bound $R_{S e g\left(\mathbb{P} A_{1} \otimes \cdots \otimes \mathbb{P} A_{n}\right.}\left(y+y^{\prime}+y^{\prime \prime}\right) \leq\left(\begin{array}{c}n+1 \\ 2\end{array}\right)$ is not tight; as for $n=3$ the following theorem shows $R_{S e g(\mathbb{P} A \times \mathbb{P} B \times \mathbb{P} C)}\left(y+y^{\prime}+y^{\prime \prime}\right)$ is at most five.

Theorem 1.8 The rank of a general point of the form $\left[y+y^{\prime}+y^{\prime \prime}\right]$ of $\sigma_{3}(\operatorname{Seg}(\mathbb{P} A \times$ $\mathbb{P} B \times \mathbb{P} C)$ ) as well as the rank of a general point of the form $\left[x^{\prime}+y^{\prime}\right]$ where $[x],[y]$ lie on a line in $\operatorname{Seg}(\mathbb{P} A \times \mathbb{P} B \times \mathbb{P} C)$, is 5 . All other points of $\sigma_{3}(\operatorname{Seg}(\mathbb{P} A \times \mathbb{P} B \times$ $\mathbb{P} C)$ ) have rank less than five, so in particular, the maximum rank of any point of $\sigma_{3}(\operatorname{Seg}(\mathbb{P} A \times \mathbb{P} B \times \mathbb{P} C))$ is 5 .

Remark 1.9 Theorem 1.8 seems to have been a "folklore" theorem in the tensor literature. For example, in Table 3.2 of [9], the result is stated and refers to [10], but in that paper the result is stated and a paper that never appeared is referred to. Also, there appear to have been privately circulating proofs; one due to R. Rocci from 1993 has been shown to us. We thank M. Mohlenkamp for these historical remarks.

The Comon conjecture on ranks says that for $T \in S^{d} V \subset V^{\otimes d}$ the symmetric tensor rank of $T$ equals the tensor rank of $T$.

Corollary 1.10 The Comon conjecture holds for $T \in \hat{\sigma}_{3}\left(v_{3}(\mathbb{P} V)\right)$.

Corollary 1.10 follows by comparing the normal forms and ranks of this paper with those of [12].

In Sect. 3 we generalize Theorem 1.2 to generalized cominuscule varieties, a class of homogeneous varieties which includes Grassmannians and spinor varieties. See Sect. 3 for the definition of a generalized cominuscule variety, and Sect. 2 for the definition of the second fundamental form $I I$.

Theorem 1.11 Let $X \subset \mathbb{P} V$ be generalized cominuscule. Then $[p] \in \sigma_{3}(X)$ if and only if at least one of the following situations occurs:

(i) $p=\xi+\eta+\zeta$ for some linearly independent $\xi, \eta, \zeta \in \hat{X}$ ( $p$ is on an honest 3-secant plane);

(ii) $p=\xi^{\prime}+\eta$ for some $\xi, \eta \in \hat{X}^{0}$ and $\xi^{\prime} \in \hat{T}_{[\xi]} X$;

(iii) $p=\xi^{\prime}+I I\left(\left(\eta^{\prime}\right)^{2}\right)$ for some $\xi \in \hat{X}, \xi^{\prime} \in \hat{T}_{[\xi]} X, \eta^{\prime} \in T_{[\xi]} X$; or

(iv) $p=\xi^{\prime}+\eta^{\prime}$ for some $\xi, \eta \in \hat{X}, \xi^{\prime} \in \hat{T}_{[\xi]} X, \eta^{\prime} \in \hat{T}_{[\eta]} X$ with the line $\mathbb{P}\langle\xi, \eta\rangle$ contained in $X$. 
To make sense of elements of the tangent and normal spaces as elements of $V$, we have chosen a splitting $V=\hat{x} \oplus T \oplus N$ as described in Sect. 2.1.

\subsection{Overview}

In Sect. 2 we review facts from projective differential geometry. In Sect. 3 we prove Theorem 1.11. In Sect. 4 we apply Theorem 1.11 to cominuscule varieties, including Grassmannians and spinor varieties. In Sect. 5 we analyze the case of the Segre variety in detail, and we give two proofs of Theorem 1.2, a short proof by computing the Lie algebras of the stabilizers of the points $p_{(*)}$, and a longer proof that contains more precise information which is of interest in its own right. In Sect. 6 we restrict attention to the three-factor Segre variety, and prove Theorems 1.3, 1.4 and 1.8.

\section{Curves in submanifolds of projective space}

2.1 Fubini forms, fundamental forms, and the prolongation property

Let $X^{n} \subset \mathbb{P} V$ be a subvariety and let $o \in X$ be a smooth point. We may choose a splitting

$$
V=\hat{o} \oplus T \oplus N
$$

such that $\hat{o} \simeq \mathbb{C}$ is the one-dimensional linear subspace corresponding to $o \in \mathbb{P} V$, and $\hat{o} \oplus T$ is the affine tangent space $\hat{T}_{o} X$.

We will abuse notation and identify $T$ with the Zariski tangent space $T_{o} X=$ $\hat{o}^{*} \otimes\left(\hat{T}_{o} X / \hat{o}\right)$ and $N$ with the normal space $N_{o} X:=T_{o} \mathbb{P} V / T_{o} X$. Since we are working at a point, the twist by the line bundle will not matter. Our choice of splitting will not affect the end results of the calculations.

Any point $[v] \in \mathbb{P} V$ has a lift to a point $v \in V$ of the form $\left(\bar{o}, \tilde{v}, v_{N}\right)$ or $\left(0, \tilde{v}, v_{N}\right)$, where 0 and $\bar{o}$ are points in $\hat{o} \simeq \mathbb{C}$, and $\tilde{v} \in T, v_{N} \in N$. In an analytic neighborhood of $o$ we may write $X$ as a graph, that is, for $x \in X$ near $o$, the vector $x_{N}$ depends holomorphically on the vector $\tilde{x}$ and we expand this holomorphic map into a Taylor series:

$$
x_{N}=x_{N}(\tilde{x})=I_{o}\left(\tilde{x}^{2}\right)+F_{3, o}\left(\tilde{x}^{3}\right)+F_{4, o}\left(\tilde{x}^{4}\right)+\cdots
$$

Here $\tilde{x} \in T$ and $\tilde{x}^{s} \in S^{s} T$. Later we will study curves $x(t) \in X$, and express the whole curve using (2.2), writing $\tilde{x}(t)$ to be the curve in $T, \tilde{x}^{s}(t) \in S^{s} T$. Note that by our choice of splitting there is no constant or linear term in (2.2). The quadratic part $I I_{o}=F_{2, o}$ gives rise to a well-defined tensor in $S^{2} T_{o}^{*} X \otimes N_{o} X \simeq S^{2} T^{*} \otimes N$, called the second fundamental form. Further, the $F_{S, o} \in S^{S} T^{*} \otimes N$ are called the Fubini forms, but they depend on the choice of splitting $V=\hat{o} \oplus T \oplus N$. See [8, Chap. 3] for more details.

One can extract tensors from the Fubini forms, called fundamental forms. Let

$$
N_{s, o}:=N_{o} X \bmod \operatorname{Image}\left(F_{2, o}, \ldots, F_{S-1, o}\right) .
$$


The tensor $\mathbb{F}_{s, o}:=\left(F_{s, o} \bmod \operatorname{Image}\left(F_{2, o}, \ldots, F_{s-1, o}\right)\right) \in S^{s} T_{o}^{*} X \otimes N_{s, o}$ is welldefined (independent of the choice of splitting (2.1)) and called the $s$-th fundamental form of $X$ at $o$. Fundamental forms satisfy a prolongation property (see [8, Chap. 3]): if $o \in X$ is a general point, then for all $f_{1} \in S^{s_{1}} T$ and $f_{2} \in S^{s_{2}} T$ we have

$$
\mathbb{F}_{s_{1}, o}\left(f_{1}\right)=0 \Longrightarrow \mathbb{F}_{s_{1}+s_{2}, o}\left(f_{1} f_{2}\right)=0 \text {. }
$$

We write $I I I_{o}=\mathbb{F}_{3, o}$. If there is no risk of confusion, we will often omit the base point and write $I I:=I I_{o}, F_{s}:=F_{s, o}$, etc.

2.2 When taking limits, we may assume one curve is stationary

Lemma 2.1 Let $G$ be a connected algebraic group and $P$ a parabolic subgroup. Let $X=G / P \subset \mathbb{P} V$ be a homogeneously embedded homogeneous variety and let $p \in \sigma_{r}(X)$. Then there exist a point $\xi \in \hat{X}$ and $r-1$ curves $y_{j}(t) \in \hat{X}$ such that $p \in \lim _{t \rightarrow 0}\left\langle\xi, y_{1}(t), \ldots, y_{r-1}(t)\right\rangle$.

Proof Since $p \in \sigma_{r}(X)$, there exist $r$ curves $x(t), y_{1}(t), \ldots, y_{r-1}(t) \in \hat{X}$ such that

$$
p \in \lim _{t \rightarrow 0}\left\langle x(t), y_{1}(t), \ldots, y_{r-1}(t)\right\rangle .
$$

Choose a curve $g_{t} \in G$, such that $g_{t}(x(t))=x_{0}=x(0)$ for all $t$ and $g_{0}=I d$. We have

$$
\begin{aligned}
\left\langle x(t), y_{1}(t), \ldots, y_{r-1}(t)\right\rangle & =g_{t}^{-1} \cdot\left\langle x_{0}, g_{t} \cdot y_{1}(t), \ldots, g_{t} \cdot y_{r-1}(t)\right\rangle \quad \text { and } \\
\lim _{t \rightarrow 0}\left\langle x(t), y_{1}(t), \ldots, y_{r-1}(t)\right\rangle & =\lim _{t \rightarrow 0}\left(g_{t}^{-1} \cdot\left\langle x_{0}, g_{t} \cdot y_{1}(t), \ldots, g_{t} \cdot y_{r-1}(t)\right\rangle\right) \\
& =\lim _{t \rightarrow 0}\left\langle x_{0}, g_{t} \cdot y_{1}(t), \ldots, g_{t} \cdot y_{r-1}(t)\right\rangle .
\end{aligned}
$$

Set $\xi=x_{0}$ and appropriately modify the $y_{j}(t)$ to complete the proof.

We remark that for non-homogeneous $X$, an analogous statement is rarely true. If $r=2$, and $X$ is smooth, then it is true, see Proposition 2.3. But already if $r=2$ and $X$ is singular, one often needs both curves moving (a cuspidical rational curve embedded in $\mathbb{P}^{3}$ is an example). Also if $r=3$, and $X$ has a trisecant line (for example, $X$ is a high degree rational normal curve projected from a general point on a trisecant plane), then one also needs three curves moving to obtain some of the points on the third secant variety.

\subsection{Dimension counting and higher order invariants}

Since $\operatorname{dim} \sigma_{r}(X) \leq r \operatorname{dim} X+r-1$, one can use a parameter count to see what one expects in choosing a point of the boundary. Suppose $\operatorname{dim} X>1, X$ is not a cone and the third fundamental form is non-zero - for example, $X=\operatorname{Seg}(\mathbb{P} A \times \mathbb{P} B \times \mathbb{P} C)$. One can predict that the third fundamental form does not arise when computing a point of $\sigma_{3}(X)$ which is on a plane obtained as a limit of spans of 3 points converging to the same general point of $X$. This is because the third fundamental form is only 
well-defined modulo the second osculating space, which will have dimension greater than $\operatorname{dim} X$. In the case of the three-factor Segre variety the second osculating space has dimension $\mathbf{a b}+\mathbf{a c}+\mathbf{b c}$, and the third fundamental form is only well-defined modulo the second osculating space. So were there a term $\operatorname{III}\left(v^{3}\right)$ appearing in an expression for a point on $\sigma_{3}(\operatorname{Seg}(\mathbb{P} A \times \mathbb{P} B \times \mathbb{P} C))$, with no restrictions on $v$, then the resulting variety would have to have dimension at least $\mathbf{a b}+\mathbf{a c}+\mathbf{b c}$ for the term to be well-defined. If the dimensions of the vector spaces are sufficiently large, this contradicts the dimension count. Such heuristics can be useful in calculations.

The following lemma will allow us to eliminate higher fundamental forms from our considerations when studying $\sigma_{3}(X)$. It illustrates the dimension counting principle.

Lemma 2.2 Let $X \subset \mathbb{P} V$ be a variety and let $o \in X$ be a general point. Adopt the notation of Sect. 2.1. Suppose $\tilde{v}(t) \subset T$ is a curve such that $I I\left(\tilde{v}(t)^{2}\right)$ vanishes at $t=0$ up to order $m-1$, that is $I I\left(\tilde{v}(t)^{2}\right)=t^{m}(\cdots)$. If $m>0$ and $s \geq 2$, then $\mathbb{F}_{s}\left(\tilde{v}(t)^{s}\right)$ vanishes at $t=0$ up to order $m+s-3$, that is $\mathbb{F}_{s}\left(\tilde{v}(t)^{s}\right)=t^{m+s-2}(\cdots)$.

Proof Let $\mathcal{I}^{d}:=\left\{f \in S^{d} T \mid \mathbb{F}_{d}(f)=0\right\}$. Since $\mathcal{I}^{d}$ is a linear subspace of $S^{d} T$, the prolongation property (2.3) implies $\mathcal{I}^{d_{1}} \cdot S^{d_{2}} T \subset \mathcal{I}^{d_{1}+d_{2}}$. Thus, if $S:=\bigoplus_{d=0}^{\infty} S^{d} T$ is the symmetric algebra, and $\mathcal{I}:=\bigoplus_{d=0}^{\infty} \mathcal{I}^{d}$, then $\mathcal{I}$ is a homogeneous ideal.

Consider $S[[t]]$, the power series ring with coefficients in $S$, and let $\mathcal{J}_{k}$ be the ideal generated by $\mathcal{I}$ and $t^{k}$. The curve $\tilde{v}=\tilde{v}(t)=\tilde{v}_{0}+t \tilde{v}_{1}+t^{2} \tilde{v}_{2}+\cdots$ is naturally an element in $S[[t]]$. In this interpretation, $\mathbb{F}_{S}\left(\tilde{v}(t)^{s}\right)=t^{k}(\cdots)$ if and only if $\tilde{v}(t)^{s} \in \mathcal{J}_{k}$. In particular, our assumptions are:

- $\tilde{v}(t)^{2} \in \mathcal{J}_{m}$, and

- the constant coefficient $\tilde{v}_{0}^{2} \in \mathcal{I}$ (because $m>0$ ), thus also $\tilde{v}_{0}^{s} \in \mathcal{I}$ for $s \geq 2$.

To show that $\tilde{v}(t)^{s} \in \mathcal{J}_{m+s-2}$ for $s \geq 2$, we argue by induction on $s$. Consider $\frac{\partial}{\partial t}\left(\tilde{v}(t)^{s}\right)=s \frac{\partial \tilde{v}}{\partial t} \tilde{v}^{s-1}$. By the inductive assumption, $\tilde{v}^{s-1} \in \mathcal{J}_{m+s-3}$, so $\frac{\partial}{\partial t}\left(\tilde{v}(t)^{s}\right) \in$ $\mathcal{J}_{m+s-3}$. Since the constant coefficient $\tilde{v}_{0}{ }^{s} \in \mathcal{I}$, it follows that $\tilde{v}(t)^{s} \in \mathcal{J}_{m+s-2}$, as claimed.

\subsection{Points on $\sigma_{2}(X)$}

We reprove the standard fact that a point on a secant variety to a smooth variety $X$ is either on $X$, on an honest secant line, or on a tangent line to $X$. The proof we present prepares the way for new results. Recall that if a point of $\sigma_{2}(X)$ is not on an honest secant line, it must arise from a point on a limiting $\mathbb{P}^{1}$ which is obtained by a curve of $\mathbb{P}^{1}$ 's, $\langle x(t), y(t)\rangle$, where $[x(0)]=[y(0)]$.

Proposition 2.3 Let $X \subset \mathbb{P} V$ be a smooth variety and let $[z] \in \sigma_{2}(X) \backslash \sigma_{2}(X)^{0}$. Then $z$ may be obtained from first-order information, that is, $z=u^{\prime}$ for some $[u] \in X$ and $u^{\prime} \in \hat{T}_{[u]} X$.

Proof There exist curves $[x(t)],[y(t)] \subset X$ with $x(0)=y(0)=\hat{o} \in o \backslash\{0\}$, such that $[z]$ may be obtained as a point of the limiting $\mathbb{P}^{1}=\mathbb{P}\left(\lim _{t \rightarrow 0}\langle x(t), y(t)\rangle\right)$. 
Consider a splitting $V=\hat{o} \oplus T \oplus N$ and the curves $\tilde{x}(t), \tilde{y}(t) \in T$ as above. Write:

$$
\begin{aligned}
& \tilde{x}(t)=\tilde{x}_{1} t+\tilde{x}_{2} t^{2}+\cdots+\tilde{x}_{k-1} t^{k-1}+\tilde{x}_{k} t^{k}+\tilde{x}_{k+1} t^{k+1}+\cdots \\
& \tilde{y}(t)=\tilde{x}_{1} t+\tilde{x}_{2} t^{2}+\cdots+\tilde{x}_{k-1} t^{k-1}+\tilde{y}_{k} t^{k}+\tilde{y}_{k+1} t^{k+1}+\cdots
\end{aligned}
$$

where $\tilde{x}_{j}, \tilde{y}_{j} \in T$ and $k$ is the smallest integer such that $\tilde{v}_{0}:=\tilde{y}_{k}-\tilde{x}_{k} \neq 0$. Let $\tilde{v}(t):=$ $t^{-k}(\tilde{y}(t)-\tilde{x}(t))=\left(\tilde{y}_{k}-\tilde{x}_{k}\right)+\left(\tilde{y}_{k+1}-\tilde{x}_{k+1}\right) t+\cdots$. Then:

$$
\begin{aligned}
y(t)-x(t)= & \left(\bar{o}+\tilde{y}(t)+I I\left(\tilde{y}(t)^{2}\right)+F_{3}\left(\tilde{y}(t)^{3}\right)+\cdots\right)-\left(\bar{o}+\tilde{x}(t)+I I\left(\tilde{x}(t)^{2}\right)\right. \\
& \left.+F_{3}\left(\tilde{x}(t)^{3}\right)+\cdots\right) \\
= & t^{k} \tilde{v}(t)+I I\left(\tilde{y}(t)^{2}-\tilde{x}(t)^{2}\right)+F_{3}\left(\tilde{y}(t)^{3}-\tilde{x}(t)^{3}\right)+\cdots \\
= & t^{k} \tilde{v}(t)+I I((\tilde{y}(t)-\tilde{x}(t))(\tilde{x}(t)+\tilde{y}(t))) \\
& +F_{3}\left((\tilde{y}(t)-\tilde{x}(t))\left(\tilde{x}(t)^{2}+\tilde{x}(t) \tilde{y}(t)+\tilde{y}(t)^{2}\right)\right)+\cdots \\
= & t^{k} \tilde{v}(t)+I I\left(t^{k} \tilde{v}(t)(\tilde{x}(t)+\tilde{y}(t))\right) \\
& +F_{3}\left(t^{k} \tilde{v}(t)\left(\tilde{x}(t)^{2}+\tilde{x}(t) \tilde{y}(t)+\tilde{y}(t)^{2}\right)\right)+\cdots
\end{aligned}
$$

Since $\tilde{x}(t)$ and $\tilde{y}(t)$ have no constant terms, we obtain:

$$
\begin{aligned}
y(t)-x(t) & =t^{k} \tilde{v}_{0}+t^{k+1}(\cdots) \quad \text { and } \\
x(t) \wedge y(t) & =x(t) \wedge(y(t)-x(t)) \\
& =(\bar{o}+t(\ldots)) \wedge\left(t^{k} \tilde{v}_{0}+t^{k+1}(\cdots)\right) \\
& =t^{k}\left(\bar{o} \wedge \tilde{v}_{0}\right)+t^{k+1}(\ldots) .
\end{aligned}
$$

Recall that $\tilde{v}_{0} \wedge \bar{o} \neq 0$. Thus the limiting affine plane $\lim _{t \rightarrow 0}\langle x(t), y(t)\rangle$ is equal to $\left\langle\bar{o}, \tilde{v}_{0}\right\rangle$.

Set $\tilde{z}(t):=t \tilde{v}(t) \in T$ and $z(t):=\bar{o}+t \tilde{v}(t)+I I\left(t^{2} \tilde{v}(t)^{2}\right)+\cdots \in \hat{X}$. Then the same affine plane can be obtained as $\lim _{t \rightarrow 0}\langle\bar{o}, z(t)\rangle$, thus one point is fixed and the other approaches the first one from the direction of $\tilde{v}_{0}$.

\section{Generalized cominuscule varieties: proof of Theorem 1.11}

Following [13], a homogeneously embedded homogeneous variety $G / P \subset \mathbb{P} V$ is called generalized cominuscule if there is a choice of splitting (at any point) such that the Fubini forms reduce to fundamental forms, that is,

$$
V=\hat{o} \oplus T \oplus N_{2} \oplus N_{3} \oplus \cdots \oplus N_{f}
$$

with $F_{S}\left(S^{s} T\right) \subset N_{s}$ and thus $F_{S}=\mathbb{F}_{s}$ for all $s \in\{2, \ldots, f\}$, and $F_{S}=\mathbb{F}_{s}=0$ for all $s>f$. Generalized cominuscule varieties may be characterized intrinsically as the homogeneously embedded $G / P$ where the unipotent radical of $P$ is Abelian. A generalized cominuscule variety is cominuscule if and only if $G$ is simple and the 
embedding is the minimal homogeneous one. For those familiar with representation theory, a homogeneously embedded homogeneous variety $G / P \subset \mathbb{P} V$ is cominuscule if $V$ is a fundamental representation $V_{\omega_{i}}$ where $\omega_{i}$ is a cominuscule weight, that is, the highest root of $\mathfrak{g}$ has coefficient one on the simple root $\alpha_{i}$. Generalized cominuscule varieties are Segre-Veronese embeddings of products of cominuscule varieties.

Grassmannians $G(k, W)$, projective spaces $\mathbb{P}^{n}$ and products of projective spaces in any homogeneous embedding (in particular, respectively, $G(k, W)$ in the Plücker embedding, Veronese varieties, and Segre varieties) are generalized cominuscule.

Throughout this section we assume $X$ is generalized cominuscule. When studying points of $\sigma_{3}(X)$, one has to take into account curves limiting to points on a trisecant line of $X$. When $X$ is cut out by quadrics, as with homogeneous varieties, any trisecant line of $X$ will be contained in $X$. Theorem 1.11 shows such points are already accounted for by curves with just one or two limit points, and that higher order differential invariants do not appear, as was hinted at in Lemma 2.2.

We commence the proof of Theorem 1.11 with an observation about the freedom of choice of splitting as in (3.1).

Lemma 3.1 Let $X$ be generalized cominuscule and let $x, y_{1}, \ldots, y_{r-1}$ be $r$ points on $X$. Then there exists a choice of splitting as in (3.1) (so $F_{S}\left(S^{s} T\right) \subset N_{S}$ for all $\left.s\right)$, such that $x=o$ is the center of this splitting and none of the points $y_{1}, \ldots, y_{r-1}$ lies on the hyperplane $T \oplus N_{2} \oplus N_{3} \oplus \cdots$.

Proof Let $G$ be the automorphism group of $X$ and $P \subset G$ be the parabolic subgroup preserving $x$. Let $Y \subset X \times \mathbb{P} V^{*}$ be the set of those pairs $(o, H)$, where $o \in X$ and $H \subset$ $V$ is a hyperplane, such that $V=\hat{o} \oplus H$ and there exists a splitting $H=T \oplus N_{2} \oplus$ $N_{3} \oplus \cdots$, making the splitting of $V$ as in (3.1). Since $X$ is generalized cominuscule, $Y$ is non-empty. It is also $G$-invariant, under the natural action $g \cdot(x, H)=(g \cdot x, g$. $H)$. Let $Y_{x} \subset \mathbb{P}\left(V^{*}\right)$ be the fiber over $x$. It is also non-empty, because $G$ acts on $X$ transitively, and it is $P$-invariant. Since the Lie algebra of $P$ contains all positive root spaces, and $\hat{x}$ is the highest weight space, the line $\hat{x}$ is contained in every $P$-invariant linear subspace of $V$ (see, e.g., [7, Prop. 14.13]).

Fix $H_{0} \in Y_{x}$ and consider the intersection $B:=\bigcap_{p \in P} p \cdot H_{0}$. This is a linear subspace of $V$, which is invariant under $P$. So either $B=0$ or $\hat{x} \subset B$. The latter is however impossible, as $\hat{x} \cap H_{0}=0$ by our assumptions. So $B=0$. The set of hyperplanes $\left\{p \cdot H_{0} \in \mathbb{P} V^{*} \mid p \in P\right\}$ is non-empty, irreducible with trivial base locus, so its dimension is positive and by a trivial instance of Bertini's Theorem there exists at least one hyperplane $H$ in this set that avoids all points $y_{1}, \ldots, y_{r-1}$.

Since there are only finitely many non-zero Fubini forms, the parameterization

$$
\begin{aligned}
\phi: T & \rightarrow \hat{X} \\
\tilde{v} & \mapsto \bar{o}+\tilde{v}+I I\left(\tilde{v}^{2}\right)+\cdots
\end{aligned}
$$

is polynomial. 
Remark 3.2 Suppose $X$ is the closure of the image of a map

$$
\begin{aligned}
\phi: T & \rightarrow \mathbb{P} V \\
\tilde{v} & \mapsto \bar{o}+\tilde{v}+v_{N}(\tilde{v})
\end{aligned}
$$

with $V=o \oplus T \oplus N, \bar{o} \in o \backslash\{0\}$, and a polynomial map $v_{N}: T \rightarrow N$. Then every point $y \in X$ is either on the hyperplane $\mathbb{P}(T \oplus N)$, or is in the image of the parameterization $\phi$.

Proof We use the following elementary topological statement: Let $P$ be a topological space, let $I \subset U \subset P$ with $I$ closed in $U$, and let $\bar{I}$ be the closure of $I$ in $P$. Then $\bar{I} \cap U=I$. To prove this, let $J \subset P$ be a closed subset such that $U \cap J=I$, which exists from the definition of subspace topology. Then $\bar{I} \subset J$, from the definition of the closure, and so

$$
I \subset \bar{I} \cap U \subset J \cap U=I .
$$

We use the statement with $P=\mathbb{P} V, U$ the affine piece of $\mathbb{P} V$, which is the complement of the hyperplane $\mathbb{P}(T \oplus N)$, and $I=\phi(T)$. Note that $\phi(T)$ is closed in $U \simeq T \oplus N$, because it is the graph of $v_{N}$ (which is a polynomial map by our assumption). Moreover, $\bar{I}=X$, and so $X \cap U=I$, and $X \subset I \cup \mathbb{P}(T \oplus N)$, as claimed.

This implies the following property of tangent spaces on $X$.

Lemma 3.3 Let $X$ be generalized cominuscule and let $\ell \subset X$ be a line. Then the space $\mathcal{T}^{\ell}:=\hat{T}_{[\xi]} X+\hat{T}_{[\eta]} X$ for any $[\xi],[\eta] \in \ell$ is independent of the choice of $[\xi],[\eta]$. Moreover, $\operatorname{dim} \mathcal{T}^{\ell}$ is constant over each irreducible component of the space parameterizing lines on $X$.

Proof Fix $o:=[\xi] \in \ell$. By Lemma 3.1 we may choose a splitting (3.1) such that $[\eta] \notin$ $T \oplus N$. Thus $[\eta]$ is in the image of the parameterization by Remark 3.2. Consider a curve $y(t) \in \hat{X}$ with $y(0)=\eta$. Note that $\tilde{y}(0) \in T$ is in the tangent direction to $\ell$. Then in the splitting (3.1),

$$
\begin{aligned}
y^{\prime}(0) & =\left.\frac{\mathrm{d}}{\mathrm{d} t}\left(\bar{o}+\tilde{y}(t)+I I\left(\tilde{y}(t)^{2}\right)+I I I\left(\tilde{y}(t)^{3}\right)+\cdots\right)\right|_{t=0} \\
& =\tilde{y}^{\prime}(0)+2 I I\left(\tilde{y}^{\prime}(0) \tilde{y}(0)\right)+3 I I I\left(\tilde{y}^{\prime}(0) \tilde{y}(0)^{2}\right)+\cdots \\
& \stackrel{(\star)}{=} \tilde{y}^{\prime}(0)+2 I I\left(\tilde{y}^{\prime}(0) \tilde{y}(0)\right) .
\end{aligned}
$$

Here $(\star)$ holds by the prolongation property $(2.3)$, because $I I\left(\tilde{y}(0)^{2}\right)=0$. Thus letting $v^{\prime}$ be any non-zero vector in $T_{\xi} \ell \subset T$ we have:

$$
\mathcal{T}^{\ell}=\hat{T}_{[\xi]} X+\hat{T}_{[\eta]} X=\left\{\xi^{\prime}+I I\left(\zeta^{\prime} v^{\prime}\right) \mid \text { for } \xi^{\prime} \in \hat{T}_{[\xi]} X, \zeta^{\prime} \in T_{[\xi]} X\right\} .
$$

This formula is independent of $\eta$, so we can vary $\eta \in \ell$ freely. Exchanging the roles of $\xi$, and $\eta$, we can also vary $\xi$. 
Thus, $\mathcal{T}^{\ell}$ is determined by the geometry of $\ell \subset X$. But the group of automorphisms of $X$ acts transitively on each irreducible component of the space parameterizing lines on $X$. When $X=G / P$ with $G$ simple, this is [11, Theorem 4.3] and [5]. (This is true for any minimally embedded homogeneous variety $G / P_{I}$, with $G$ simple, where $I$ indexes the deleted simple roots, as long as $I$ does not contain an "exposed short root" in the language of [11].) When $X=\operatorname{Seg}\left(v_{d_{1}}\left(G_{1} / P_{1}\right) \times \cdots \times\right.$ $v_{d_{n}}\left(G_{n} / P_{n}\right)$ ) is generalized cominuscule (with each $G_{i} / P_{i}$ cominuscule), the set of lines on $X$ is the disjoint union of the variety of lines on each $G_{i} / P_{i}$ such that $d_{i}=1$. Thus $\operatorname{dim} \mathcal{T}^{\ell}$ must be constant over these irreducible components.

Lemma 3.3 allows an alternative interpretation of the points of type (iv):

Lemma 3.4 With the notation as in Theorem 1.11 , let $\mathrm{Z}(X)$ denote the set of points of type (iv). Then $[p] \in \mathrm{Z}(X)$ if and only if

(iv') $p=\xi^{\prime}+I I\left(\zeta^{\prime} v^{\prime}\right)$ for some $\xi \in \hat{X}, \xi^{\prime} \in \hat{T}_{[\xi]} X, \zeta^{\prime}, v^{\prime} \in T_{[\xi]} X$ with $I I\left(\left(v^{\prime}\right)^{2}\right)=0$, i.e., $v^{\prime}$ is tangent to a line on $X$ through $\xi$.

Furthermore, $\mathrm{Z}(X)$ is a closed subset of $\mathbb{P} V$.

Proof The alternative description (iv') follows from (3.2).

To see that $\mathrm{Z}(X)$ is a closed subset of $\mathbb{P} V$, note $\mathrm{Z}(X)$ is the image of a projective space bundle over the variety parameterizing lines on $X$, whose fiber over $\ell \subset X$ is $\mathbb{P}\left(\mathcal{T}^{\ell}\right)$. Since $\operatorname{dim} \mathcal{T}^{\ell}$ is locally constant by Lemma 3.3 , this bundle is a projective variety, and thus $\mathrm{Z}(X)$ is an image of a projective variety, hence projective.

In the following lemma, we provide an uniform interpretation of the points of types (iii)-(iv).

Lemma $3.5[p]$ is of type (iii) or (iv), if and only if

(iii-iv) $p=\xi^{\prime}+u$ for some $\xi \in \hat{X}^{0}, \xi^{\prime} \in \hat{T}_{[\xi]} X$, and $u \in \overline{I I}:=\overline{\left\{I I\left(\tilde{v}^{2}\right): \tilde{v} \in T\right\}}$.

Moreover, for $u \in V$, the following conditions are equivalent:

(1) $u \in \overline{I I}$;

(2) There exist a curve $\tilde{v}(t) \in T$ and an integer $m$, such that $I I\left(\tilde{v}(t)^{2}\right)=t^{m} u+$ $t^{m+1}(\ldots)$;

(3) There exist an integer $m$ and vectors $\tilde{v}_{0}, \tilde{v}_{1}, \ldots, \tilde{v}_{m} \in T$, such that

$$
I I\left(\sum_{i=0}^{d} \tilde{v}_{i} \tilde{v}_{d-i}\right)=\left\{\begin{array}{ll}
0 & \text { if } d<m \\
u & \text { if } d=m
\end{array} .\right.
$$

Note that $\mathbb{P} \overline{I I}$ is the closure of the image of the rational map $i i: \mathbb{P} T \rightarrow \mathbb{P} N$ given by $[\tilde{v}] \mapsto\left[I I\left(\tilde{v}^{2}\right)\right]$.

Proof of Lemma 3.5 The equivalence of (1)-(3) is clear. In the notation of (3), a point $p$ is of type (iii) if and only if it is of type (iii-iv) with $m=0$, and it is of type (iv) 
if and only if it is of type (iii-iv) with $m=1$. So suppose $p$ is of type (iii-iv) with $m>1$. Then it is in the closure of $\mathrm{Z}(X)$, the set of points of type (iv). But $\mathrm{Z}(X)$ is closed by Lemma 3.4, so $p$ is of type (iv).

Proof of Theorem 1.11 Suppose $p \in \sigma_{3}(X)$, so there exist $\xi$ and $y(t):=y_{1}(t), z(t):=$ $y_{2}(t)$ as in Lemma 2.1. Write $\xi=\bar{o}$, and by Lemma 3.1 we may choose the splitting (3.1) such that for small values of $t$, we have $y(t), z(t) \notin T \oplus N$. So $y(t)=\left(\bar{o}, \tilde{y}(t), y_{N}(t)\right)$ by Remark 3.2 and similarly for $z(t)$. Consider the curves $\tilde{y}(t), \tilde{z}(t) \in T$. Exchanging the roles of $y$ and $z$ if necessary, pick maximal integers $k, l$, with $l \geq k \geq 0$ and such that

$$
\begin{aligned}
& \tilde{y}(t)=t^{k} \tilde{v}(t) \quad \text { and } \\
& \tilde{z}(t)=t^{k} \lambda(t) \tilde{v}(t)+t^{l} \tilde{w}(t)
\end{aligned}
$$

for some holomorphic function $\lambda(t) \in \mathbb{C}$ and curves $\tilde{v}(t), \tilde{w}(t) \in T$. From now on, we write $y$ for $y(t)$, etc. We adopt the convention $l=\infty$ if $\tilde{w}=0$.

If $l=0$, then $0, \tilde{y}_{0}, \tilde{z}_{0}$ are three distinct and non-collinear points in $T$. This implies that $p$ is on an honest 3-secant, and we are in case (i). So from now on suppose $l>0$.

Our goal is to understand the leading term (in $t$ ) of

$$
\bar{o} \wedge y \wedge z=\bar{o} \wedge(y-\bar{o}) \wedge(z-\bar{o}-\lambda(y-\bar{o})) .
$$

Expanding out terms we obtain:

$$
\begin{aligned}
y-\bar{o} & =t^{k} \tilde{v}+t^{2 k} I I\left(\tilde{v}^{2}\right)+t^{3 k} I I I\left(\tilde{v}^{3}\right)+\cdots \\
(z-\bar{o} & -\lambda(y-\bar{o})) \\
& =t^{l} \tilde{w}+\sum_{s=2}^{f} \mathbb{F}_{s}\left(\tilde{z}^{s}-\lambda \tilde{y}^{s}\right) \\
& =t^{l} \tilde{w}+\sum_{s=2}^{f} \mathbb{F}_{s}\left(\left(\lambda t^{k} \tilde{v}+t^{l} \tilde{w}\right)^{s}-\lambda t^{s k} \tilde{v}^{s}\right) \\
& =t^{l} \tilde{w}_{0}+\sum_{s=2}^{f} \mathbb{F}_{s}\left(\left(\lambda^{s}-\lambda\right) t^{s k} \tilde{v}^{s}+s \lambda^{s-1} t^{(s-1) k+l} \tilde{v}^{s-1} \tilde{w}\right)+t^{l+1}(\ldots) \\
& =t^{l} \tilde{w}_{0}+\sum_{s=2}^{f}\left(\lambda^{s}-\lambda\right) t^{s k} \mathbb{F}_{s}\left(\tilde{v}^{s}\right)+s \lambda^{s-1} t^{(s-1) k+l} \mathbb{F}_{s}\left(\tilde{v}^{s-1} \tilde{w}\right)+t^{l+1}(\ldots) .
\end{aligned}
$$

First consider the case $k \geq 1$, so that the three limit points coincide: $\bar{o}=y_{0}=z_{0}$. In this case, the terms in (3.4) with $t^{(s-1) k+l}$ are of order higher than $l$. By Lemma 2.2, the higher fundamental forms $\mathbb{F}_{s}$ with $s \geq 3$ will always have higher degree leading term than II. Thus:

$$
\bar{o} \wedge y \wedge z=\bar{o} \wedge t^{k} \tilde{v}_{0} \wedge\left(t^{l} \tilde{w}_{0}+t^{2 k} \lambda(\lambda-1) I I\left(\tilde{v}^{2}\right)\right)+\cdots \text { terms of higher order. }
$$


We conclude that any point $p$ in the limiting space, which is spanned by $\bar{o}, \tilde{v}_{0}$, and the leading term of $\left(t^{l} \tilde{w}_{0}+t^{2 k} \lambda(\lambda-1) I I\left(\tilde{v}^{2}\right)\right)$, is of the form (iii-iv).

In the remainder of the argument assume $k=0$ and we still assume $l>0$.

If $\lambda_{0} \neq 0,1$, the three limit points $0, \tilde{y}_{0}, \tilde{z}_{0}$ are distinct, but they lie on a line in $T$. Also suppose that $I I\left(\tilde{v}_{0}^{2}\right) \neq 0$. This means (e.g. by (2.2)) that the projective line from $o$ in the direction of $\tilde{v}_{0}$ is not contained in $X$. It follows that $\bar{o}, y_{0}, z_{0}$ are linearly independent, because any line trisecant to $X$ is entirely contained in $X$. This leads to case (i).

Now, say $\lambda_{0}=0$ or 1 , and $I I\left(\tilde{v}_{0}^{2}\right) \neq 0$. If $\lambda_{0}=0$, then $\bar{o}=z(0)$. If $\lambda_{0}=1$, then $y(0)=z(0)$. Swapping the roles of $x$ and $y$ if necessary, we may assume $\lambda_{0}=0$ and write $\lambda=t^{m} \lambda_{m}+t^{m+1}(\ldots)$ with $m \geq 1$ and $\lambda_{m} \neq 0$. Note also $\tilde{y}=\tilde{v}$ in this case (because $k=0$ ). Then the leading term of (3.4) is the leading term of $t^{l} \tilde{w}_{0}+$ $\sum_{s=2}^{f}\left(\lambda^{s}-\lambda\right) \mathbb{F}_{s}\left(\tilde{y}_{0}^{s}\right)$ or it is of order at least $l+1$. Therefore:

$$
\begin{aligned}
\bar{o} \wedge y \wedge z= & \bar{o} \wedge y_{0} \wedge\left(t^{l} \tilde{w}_{0}+\sum_{s=2}^{f}\left(\lambda^{s}-\lambda\right) \mathbb{F}_{s}\left(\tilde{y}_{0}^{s}\right)\right)+\text { terms of higher order } \\
= & \bar{o} \wedge y_{0} \wedge(t^{l} \tilde{w}_{0}+\underbrace{\sum_{s=2}^{f} \lambda^{s} \mathbb{F}_{s}\left(\tilde{y}_{0}^{s}\right)}_{=t^{2 m} \cdot(\ldots)}-\lambda \underbrace{\sum_{s=2}^{f} \mathbb{F}_{s}\left(\tilde{y}_{0}^{s}\right)}_{=y_{0}-\bar{o}-\tilde{y}_{0}}) \\
& + \text { terms of higher order } \\
= & \bar{o} \wedge y_{0} \wedge\left(\lambda \tilde{y}_{0}+t^{l} \tilde{w}_{0}\right)+\text { terms of higher order } \\
= & \bar{o} \wedge y_{0} \wedge\left(\lambda_{m} t^{m} \tilde{y}_{0}+t^{l} \tilde{w}_{0}\right)+\text { terms of higher order. }
\end{aligned}
$$

Note $y_{0}$ is linearly independent from $T$, because $I I\left(\tilde{y}_{0}^{2}\right) \neq 0$. We cannot have $m=l$ and $\tilde{w}_{0}=-\lambda_{m} \tilde{y}_{0}$, because then the choice of $l$ would not be maximal. Thus we have non-zero terms of degrees $l$ or $m$, and the limiting space is spanned by $\bar{o}, y_{0}$ and a tangent vector to $o$ (which is a linear combination of $\tilde{y}_{0}$ and $\tilde{w}_{0}$ ). Therefore we are in case (ii).

Finally, suppose $I I\left(\tilde{v}_{0}^{2}\right)=0$ (so the line $\langle o, y(0)\rangle$ is contained in $\left.X\right)$.

Hence (3.4) becomes

$$
t^{l} \tilde{w}_{0}+\sum_{s=2}^{f}\left(\left(\lambda^{s}-\lambda\right) \mathbb{F}_{s}\left(\tilde{v}^{s}\right)+s \lambda^{s-1} t^{l} \mathbb{F}_{s}\left(\tilde{v}^{s-1} \tilde{w}\right)\right)+t^{l+1}(\ldots) .
$$

We claim that the summands with $\mathbb{F}_{s}$ for $s \geq 3$ are irrelevant to the leading term. First note for $s \geq 3$ the fundamental form $\mathbb{F}_{s}\left(\tilde{v}^{s-1} \tilde{w}\right)$ vanishes at $t=0$ by the prolongation property (2.3). So $t^{l} \mathbb{F}_{s}\left(\tilde{v}^{s-1} \tilde{w}\right)$ has order of vanishing at least $l+1$, unless $s=2$. Next we treat

$$
\sum_{s=2}^{f}\left(\lambda^{s}-\lambda\right) \mathbb{F}_{s}\left(\tilde{v}^{s}\right)=\left(\lambda^{2}-\lambda\right) \sum_{s=2}^{f}\left(1+\lambda+\cdots+\lambda^{s-2}\right) \mathbb{F}_{s}\left(\tilde{v}^{s}\right)
$$


By Lemma 2.2, for $s \geq 3$ the leading term of $\mathbb{F}_{s}\left(\tilde{v}^{s}\right)$ is of higher order than that of $I I\left(\tilde{v}^{s}\right)$. Thus the leading term of (3.4) can only come from the leading term of

$$
t^{l} \tilde{w}_{0}+\left(\lambda^{2}-\lambda\right) I I\left(\tilde{v}^{2}\right)+2 \lambda t^{l} I I\left(\tilde{v} \tilde{w}_{0}\right) .
$$

Suppose $\mu$ is a holomorphic function in one variable, and $m$ is the maximal integer such that $\lambda-1=t^{m} \mu^{2}$ for sufficiently small values of $t$. Note that $\mu$ has invertible values near $t=0$. If $m \geq l$, then only $t^{l} \tilde{w}_{0}+2 \lambda t^{l} I I\left(\tilde{v} \tilde{w}_{0}\right)$ contributes to the leading term of (3.4), and $p$ is of type (iii-iv). Suppose $m<l$, and rewrite (3.5), up to terms of order $>l$ :

$$
t^{l} \tilde{w}_{0}+\lambda t^{m} I I\left(\left(\mu \tilde{v}+\frac{t^{l-m}}{\mu} \tilde{w}_{0}\right)^{2}\right) .
$$

Thus there exists $u \in \overline{I I}$ (either $u=0$ or $u$ is the leading coefficient of $I I((\mu \tilde{v}+$ $\left.\frac{t^{l-m}}{\mu} \tilde{w}_{0}\right)^{2}$ ) up to scale, compare with Lemma 3.5(2)), such that the limiting space $\lim _{t \rightarrow 0}\langle\bar{o}, y(t), z(t)\rangle$ is spanned by either $\bar{o}, y_{0}, u$ or $\bar{o}, y_{0}, \tilde{w}_{0}+u$. Since $y_{0} \in \hat{o} \oplus T$, in either case we have $p=\xi^{\prime}+u$ for some $\xi^{\prime} \in \hat{o} \oplus T$, a linear combination of $\bar{o}, y_{0}$ and $\tilde{w}_{0}$, and also after possible rescaling of $u$. That is, $p$ is a point of type (iii-iv).

It remains to prove that any point $p$ of the form (i), (ii), or (iii-iv) is in $\sigma_{3}(X)$. Case (i) is clear, case (ii) follows as $\sigma_{3}(X)=J\left(X, \sigma_{2}(X)\right) \supset J(X, \tau(X))$ and points on tangent lines are handled by Proposition 2.3.

Finally, for case (iii-iv), take $\xi=\bar{o}$, and $\xi^{\prime}=\bar{o}+\tilde{w}_{0}$ with $\tilde{w}_{0} \in T$. For $u \in \overline{I I}$, let $\tilde{v}$ and $m$ be as in Lemma 3.5(2). Set:

$$
\begin{aligned}
& x(t):=\bar{o} \\
& y(t):=\bar{o}+t \tilde{v}+t^{2} I I\left(\tilde{v}^{2}\right)+\cdots, \quad \text { and } \\
& z(t):=\bar{o}+2 t \tilde{v}+4 t^{2} I I\left(\tilde{v}^{2}\right)+\cdots+2 t^{m+2} \tilde{w}_{0}+\cdots
\end{aligned}
$$

i.e. $\tilde{y}(t)=t \tilde{v}$ and $\tilde{z}(t)=2 t \tilde{v}+2 t^{m+2} \tilde{w}_{0}$. We calculate:

$x(t) \wedge y(t) \wedge z(t)=\bar{o} \wedge t \tilde{v} \wedge t^{m+2}\left(2 \tilde{w}_{0}+2 u\right)+\cdots$ terms of higher order.

Here $\xi^{\prime}+u=\bar{o}+\tilde{w}_{0}+u$ is in the limiting space.

\section{Examples}

In the next sections we treat the case of Segre product with at least 3 factors in detail. Here we briefly review some other cases.

\subsection{Known results}

We record the following known results:

Example 4.1 Let $X \subset \mathbb{P} V$ be one of $v_{2}\left(\mathbb{P}^{n}\right)$ (symmetric matrices of rank one), $G(2, n)$ (skew-symmetric matrices of rank two), $\operatorname{Seg}(\mathbb{P} A \times \mathbb{P} B)$ (matrices of rank 
one), or the Cayley plane $\mathbb{O P}^{2}$. Then any point on $\sigma_{r}(X)$ for any $r$ is on an honest secant $\mathbb{P}^{r-1}$.

Example 4.2 [12] Let $X=v_{d}\left(\mathbb{P}^{n}\right)$ for $d>2$. Then any point in $\sigma_{3}(X)$ is of the form:

(i) $p=\xi+\eta+\zeta$ for some $\xi, \eta, \zeta \in \hat{X}$ ( $p$ is on an honest 3-secant plane), or

(ii) $p=\xi^{\prime}+\eta$ for some $\xi, \eta \in \hat{X}$ and $\xi^{\prime} \in T_{[\xi]} X$, or

(iii) $p=\xi^{\prime}+I I\left(\eta^{\prime}, \eta^{\prime}\right)$ for some $\xi \in \hat{X}, \xi^{\prime}, \eta^{\prime} \in T_{[\xi]} X$.

Normal forms for $\sigma_{3}\left(v_{d}(\mathbb{P} V)\right) \backslash \sigma_{2}\left(v_{d}(\mathbb{P} V)\right)$ of these types are respectively $x^{d}+y^{d}+$ $z^{d}, x^{d-1} y+z^{d}$ and $x^{d-1} y+x^{d-2} z^{2}$, where $x, y, z \in V$. Thus the points of type (iv) do not occur in this case.

The generalized cominuscule varieties with $\sigma_{2}(X)=\mathbb{P} V$ are $\operatorname{Seg}\left(\mathbb{P}^{1} \times \mathbb{P}^{n}\right)$, $\operatorname{Seg}\left(\mathbb{P}^{1} \times \mathbb{P}^{1} \times \mathbb{P}^{1}\right)$, quadric hypersurfaces $Q$, the Veronese varieties $v_{2}\left(\mathbb{P}^{1}\right), v_{3}\left(\mathbb{P}^{1}\right)$, the Grassmannians $G(2,5)$ and $G(3,6)$, the spinor varieties $\mathbb{S}_{5}$ and $\mathbb{S}_{6}$, the Lagrangian Grassmannian $G_{\text {Lag }}(3,6), \operatorname{Seg}\left(\mathbb{P}^{1} \times Q\right)$, and the Freudenthal variety $E_{7} / P_{7}$.

\subsection{Grassmannians in Plücker embedding}

Let $X:=G(k, n) \subset \mathbb{P}\left(\bigwedge^{k} \mathbb{C}^{n}\right)$, and suppose $3 \leq k \leq n-k$ and $n-k>3$. The tangent space at $E \in G(k, n)$ can be identified with the space of $k \times(n-k)$-matrices $\bigwedge^{k-1} E \otimes F \simeq E^{*} \otimes F$, where $F=\mathbb{C}^{n} / E$. The local parameterization in this case comes from a choice of splitting $\mathbb{C}^{n} \simeq E \oplus F$ and the determined splitting:

$$
\begin{aligned}
\bigwedge^{k}(E \oplus F) & =\bigwedge^{k} E \oplus \bigwedge^{k-1} E \otimes F \oplus \bigwedge^{k-2} E \otimes \bigwedge^{2} F \oplus \cdots \oplus \bigwedge^{k} F \\
& \simeq \hat{o} \oplus E^{*} \otimes F \oplus \bigwedge^{2} E^{*} \otimes \bigwedge^{2} F \oplus \cdots \oplus \bigwedge^{k} E^{*} \otimes \bigwedge^{k} F
\end{aligned}
$$

The parameterization has the following form:

$$
T \simeq E^{*} \otimes F \ni M \mapsto[\underbrace{1,}_{\in \hat{o}} \underbrace{M,}_{\in T} \underbrace{\bigwedge^{2} M,}_{=I I\left(M^{2}\right) \in N_{2}} \cdots, \underbrace{\bigwedge^{k} M}_{\in N_{k}}],
$$

where $\mathbb{F}_{s}\left(M^{s}\right)=\bigwedge^{s} M \in \bigwedge^{s} E^{*} \otimes \bigwedge^{s} F$, expressed in linear coordinates, is the collection of all $s \times s$ minors of $M$.

In the normal forms of Theorem 1.11 we can take the first point $\xi=\bar{o}$, for the second we have $k$ choices given the rank of $M$. Let $\epsilon_{i}$ for $i \in\{1, \ldots, k\}$ denote the matrix of rank $i$ with the block form $\left(\begin{array}{rr}\operatorname{Id}_{i} & 0 \\ 0 & 0\end{array}\right)$. The normal forms are:

(i) $p=\bar{o}+\varphi\left(\epsilon_{i}\right)+\varphi(M)$ for some $i, M$,

(ii) $p=\bar{o}+M+\varphi\left(\epsilon_{i}\right)$ or $p=M+\varphi\left(\epsilon_{i}\right)$ for some $i, M$,

(iii) $p=\bar{o}+M+\bigwedge^{2} \epsilon_{i}$ or $p=M+\bigwedge^{2} \epsilon_{i}$ for some $i, M$,

(iv') $p=\bar{o}+M+\bigwedge^{2} \epsilon_{i+1}-\bigwedge^{2} \epsilon_{i}$ or $p=M+\bigwedge^{2} \epsilon_{i+1}-\bigwedge^{2} \epsilon_{i}$ for some $i \neq k, M$. 
In (iv'), $v=\epsilon_{i+1}-\epsilon_{i}$ is a rank-one matrix, so $I I\left(v^{2}\right)=0$, and $\bigwedge^{2} \epsilon_{i+1}-\bigwedge^{2} \epsilon_{i}=$ $\frac{1}{2} I I\left(u, \epsilon_{i}\right)$. In all normal forms, we can pick $M$ to be in some normal form. For example, if $i=k=n-k$, then $M$ may be (at least) assumed to be in Jordan normal form.

\subsection{Lagrangian Grassmannians}

Let $X$ be the Lagrangian Grassmannian $G_{L a g}(k, 2 k)=C_{k} / P_{k} \subset \mathbb{P}\left(V_{\omega_{k}}\right)$ with $k>3$, where $V_{\omega}=\bigwedge^{k} \mathbb{C}^{2 k} / \bigwedge^{k-2} \mathbb{C}^{2 k}$ is the minimal homogeneous embedding. In this case the local parameterization is identical, but with $T \simeq S^{2} \mathbb{C}^{k}$ and $M$ a symmetric $k \times k$ matrix (see $[1, \S 5])$. The normal forms are also identical.

\subsection{Spinor varieties}

Let $X$ be the spinor variety $\mathbb{S}_{k}=D_{k} / P_{k}$ for $k \geq 7$ in its minimal homogeneous embedding $\mathbb{P}\left(\bigwedge^{\text {even }} \mathbb{C}^{k}\right)$. In this case $T \simeq \bigwedge^{2} \mathbb{C}^{k}$ and $M$ is a skew-symmetric $k \times k$ matrix, and the parameterization is similar to the previous cases:

$$
M \stackrel{\varphi}{\mapsto}[\underbrace{1,}_{\in \hat{o}} \underbrace{M,}_{\in T} \underbrace{\operatorname{Pf}_{4} M,}_{=I I\left(M^{2}\right) \in N_{2}} \underbrace{\operatorname{Pf}_{6} M,}_{I I I\left(M^{3}\right) \in N_{3}} \ldots],
$$

where $\operatorname{Pf}_{2 s} M \in \bigwedge^{2 s} \mathbb{C}^{k}$, expressed in linear coordinates, is the collection of all $2 s \times$ 2s sub-Pfaffians of $M$.

Let $\epsilon_{i}^{\text {skew }}$ for $i \in\left\{1, \ldots,\left\lfloor\frac{1}{2} k\right\rfloor\right\}$ denote the matrix of rank $2 i$ with the block form

$$
\left(\begin{array}{ccc}
0 & \mathrm{Id}_{i} & 0 \\
-\mathrm{Id}_{i} & 0 & 0 \\
0 & 0 & 0
\end{array}\right)
$$

The normal forms are:

(i) $p=\bar{o}+\varphi\left(\epsilon_{i}^{\text {skew }}\right)+\varphi(M)$ for some $i, M$,

(ii) $p=\bar{o}+M+\varphi\left(\epsilon_{i}^{\text {skew }}\right)$ or $p=M+\varphi\left(\epsilon_{i}^{\text {skew }}\right)$ for some $i, M$,

(iii) $p=\bar{o}+M+\mathrm{Pf}_{4} \epsilon_{i}^{\text {skew }}$ or $p=M+\mathrm{Pf}_{4} \epsilon_{i}^{\text {skew }}$ for some $i, M$,

(iv') $p=\bar{o}+M+\mathrm{Pf}_{4} \epsilon_{i+1}^{\text {skew }}-\mathrm{Pf}_{4} \epsilon_{i}^{\text {skew }}$ or $p=M+\mathrm{Pf}_{4} \epsilon_{i+1}^{\text {skew }}-\mathrm{Pf}_{4} \epsilon_{i}^{\text {skew }}$ for some $i \neq\left\lfloor\frac{1}{2} k\right\rfloor, M$.

\section{The Segre product $\operatorname{Seg}\left(\mathbb{P} A_{1} \times \cdots \times \mathbb{P} A_{n}\right)$}

Recall that for any smooth variety $X$, if $x \in \sigma_{2}(X)$, then either $x \in X, x \in \sigma_{2}^{0}(X)$ or $x$ lies on an embedded tangent line to $X$, see Proposition 2.3. 


\subsection{Proof of Proposition 1.1}

All the assertions except for the rank of $x$ in (1.1) are immediate. The rank of $x$ is at most $|J|$ because there are $|J|$ terms in the summation.

Assume without loss of generality $|J|=n$ and work by induction. The case $n=2$ is clear. Now assume we have established the result up to $n-1$, and consider $x\left(A_{1}^{*}\right)$. It is spanned by

$$
a_{1}^{2} \otimes \cdots \otimes a_{1}^{n}, \sum_{j=2}^{n} a_{1}^{2} \otimes \cdots \otimes a_{1}^{j-1} \otimes a_{2}^{j} \otimes a_{1}^{j+1} \otimes \cdots \otimes a_{1}^{n} .
$$

By induction, the second vector has rank $n-1$, so the only way $x\left(A_{1}^{*}\right)$ could be spanned by $n-1$ rank-one elements would be if there were an expression of the second vector as a sum of $n-1$ decomposable tensors where one of terms is a multiple of $a_{1}^{2} \otimes \cdots \otimes a_{1}^{n}$. Say there were such an expression, where $a_{1}^{2} \otimes \cdots \otimes a_{1}^{n}$ appeared with coefficient $\lambda$, then the tensor $\sum_{j=2}^{n} a_{1}^{2} \otimes \cdots \otimes a_{1}^{j-1} \otimes a_{2}^{j} \otimes a_{1}^{j+1} \otimes \cdots \otimes a_{1}^{n}-$ $\lambda a_{1}^{2} \otimes \cdots \otimes a_{1}^{n}$ would have rank $n-2$, but setting $\tilde{a}_{2}^{2}=a_{2}^{2}-\lambda a_{1}^{2}$ and $\tilde{a}_{2}^{j}=a_{2}^{j}$ for $j \in\{3, \ldots, n\}$, this would imply that

$$
\sum_{j=2}^{n} a_{1}^{2} \otimes \cdots \otimes a_{1}^{j-1} \otimes \tilde{a}_{2}^{j} \otimes a_{1}^{j+1} \otimes \cdots \otimes a_{1}^{n}
$$

had rank $n-2$, a contradiction.

Remark 5.1 The case $n=3$ was previously established by Grigoriev, Ja'Ja' and Teichert.

\subsection{Parameterization in the Segre case}

Suppose $X=\operatorname{Seg}\left(\mathbb{P} A_{1} \times \cdots \times \mathbb{P} A_{n}\right)$. Let $\bar{o}=a_{1}^{1} \otimes \cdots \otimes a_{1}^{n}$, and let $A_{j}^{\prime}=a_{1}^{1} \otimes \cdots \otimes$ $a_{1}^{j-1} \otimes\left(A_{j} / a_{1}^{j}\right) \otimes a_{1}^{j+1} \otimes \cdots \otimes a_{1}^{n} \simeq A_{j} / a_{1}^{j}$. Then $T=A_{1}^{\prime} \oplus \cdots \oplus A_{n}^{\prime}$ and $X$ is parameterized by

$$
\left(a_{1}^{\prime}, \ldots, a_{n}^{\prime}\right) \mapsto[\underbrace{1,}_{\in \hat{o}} \underbrace{a_{1}^{\prime}, \ldots, a_{n}^{\prime}}_{\in T}, \underbrace{a_{1}^{\prime} \otimes a_{2}^{\prime}, \ldots, a_{n-1}^{\prime} \otimes a_{n}^{\prime}}_{=I I\left(\left(a_{1}^{\prime}, \ldots, a_{n}^{\prime}\right)^{2}\right) \in N_{2}}, \ldots, \underbrace{a_{1}^{\prime} \otimes a_{2}^{\prime} \otimes \cdots \otimes a_{n}^{\prime}}_{\in N_{n}}] .
$$

Thus $I I\left(\left(a_{1}^{\prime}, \ldots, a_{n}^{\prime}\right) \cdot\left(b_{1}^{\prime}, \ldots, b_{n}^{\prime}\right)\right)=\frac{1}{2}\left(a_{1}^{\prime} \otimes b_{2}^{\prime}+b_{1}^{\prime} \otimes a_{2}^{\prime}, \ldots, a_{n-1}^{\prime} \otimes b_{n}^{\prime}+b_{n-1}^{\prime} \otimes\right.$ $\left.a_{n}^{\prime}\right)$.

In this case the base locus of $I I$ is $\mathbb{P} A_{1}^{\prime} \sqcup \cdots \sqcup \mathbb{P} A_{n}^{\prime} \subset \mathbb{P}\left(A_{1}^{\prime} \oplus \cdots \oplus A_{n}^{\prime}\right) \simeq \mathbb{P} T$. If $I I\left(\tilde{v}_{0}^{2}\right)=0$, then $\tilde{v}_{0} \in A_{i}^{\prime}$ for some $i$ and if further $I I\left(\tilde{v}_{0} \tilde{v}_{1}\right)=0$ then $\tilde{v}_{1} \in A_{i}^{\prime}$ for the same $i$.

In particular, if a line $\ell \subset X$ contains $o$ and is tangent to $\tilde{v}_{0}$, then by (3.2) we have:

$$
\operatorname{dim} \mathcal{T}^{\ell}=2 \operatorname{dim} X+1-\operatorname{dim} \operatorname{ker} I I\left(\tilde{v}_{0} \cdot\right)=2 \operatorname{dim} X+2-\operatorname{dim} A_{i} .
$$


Now we prove Theorem 1.2. The normal forms follow from the discussion in the previous sections.

Now suppose $\operatorname{dim} A_{i} \geq 3$. To see that the general points of each type do not belong to the other types, note that for any type and for any $i$, in the normal forms (1.2)(1.5) either $a_{1}^{i}, a_{2}^{i}, a_{3}^{i}$ are linearly independent, or the point is contained in a subspace variety, i.e., a closed subvariety consisting of tensors in some $A_{1} \otimes \cdots \otimes A_{i-1} \otimes \mathbb{C}^{2} \otimes$ $A_{i+1} \otimes \cdots \otimes A_{n}$. Thus the general points of each type form a single orbit (or $n$ orbits for type (iv)) of the action of $G L\left(A_{1}\right) \times \cdots \times G L\left(A_{n}\right)$. Therefore the only possible way that they could overlap, is if one of the orbits were equal to the other. But the orbits are distinct by the dimension count below, which we present in two different forms.

\subsection{First proof of dimensions in Theorem 1.2}

We compute the Lie algebras of the stabilizers of each type of point. Without loss of generality (for computing codimension), assume $\operatorname{dim} A_{j}=3$. Write $\Gamma=\left(x_{1}, \ldots, x_{n}\right)$ where $x_{\alpha}=\left(x_{j, \alpha}^{i}\right), 1 \leq i, j \leq 3$. We calculate the $\Gamma$ such that $\Gamma \cdot p_{(*)}=0$ in each case $*=\mathrm{i}$, ii, iii, iv and denote this algebra by $\mathfrak{g}_{p_{(*)}}$. In each case one has a system of $3^{n}=\operatorname{dim}\left(A_{1} \otimes \cdots \otimes A_{n}\right)$ linear equations, many of which are zero or redundant.

$$
\mathfrak{g}_{p_{(\mathrm{i})}}=\left\{\underset{\alpha=1, \ldots, n}{\times}\left(\begin{array}{ccc}
x_{1, \alpha}^{1} & 0 & 0 \\
0 & x_{2, \alpha}^{2} & 0 \\
0 & 0 & x_{3, \alpha}^{3}
\end{array}\right) \mid \sum_{\alpha} x_{i, \alpha}^{i}=0, i=1,2,3\right\} .
$$

Note $\operatorname{dim} \mathfrak{g}_{p_{(\mathrm{i})}}=3 n-3$.

$$
\mathfrak{g}_{p_{\text {(ii) }}}=\left\{\underset{\alpha=1, \ldots, n}{\times}\left(\begin{array}{ccc}
x_{1, \alpha}^{1} & x_{2, \alpha}^{1} & 0 \\
0 & -\sum_{\beta \neq \alpha} x_{1, \beta}^{1} & 0 \\
0 & 0 & x_{3, \alpha}^{3}
\end{array}\right) \mid \sum_{\alpha} x_{3, \alpha}^{3}=0, \sum_{\alpha} x_{2, \alpha}^{1}=0\right\} .
$$

Note $\operatorname{dim} \mathfrak{g}_{p_{\text {(ii) }}}=3 n-2$.

$$
\mathfrak{g}_{p_{(\mathrm{iii})}}=\left\{\underset{\alpha=1, \ldots, n}{\times}\left(\begin{array}{ccc}
x_{1, \alpha}^{1} & x_{2, \alpha}^{1} & x_{3, \alpha}^{1} \\
0 & -\sum_{\beta \neq \alpha} x_{1, \beta}^{1} & -\sum_{\beta \neq \alpha} x_{2, \beta}^{1} \\
0 & 0 & -\sum_{\beta \neq \alpha} x_{1, \beta}^{1}
\end{array}\right) \mid \sum_{\alpha} x_{3, \alpha}^{1}=0\right\} .
$$

Note $\operatorname{dim} \mathfrak{g}_{p_{\text {(iii) }}}=3 n-1$.

$$
\begin{aligned}
\mathfrak{g}_{p_{\text {(iv) }}} & \left\{\left(\begin{array}{ccc}
x_{1,1}^{1} & x_{2,1}^{1} & x_{3,1}^{1} \\
x_{1,1}^{2} & x_{2,1}^{2} & -\sum_{\rho} x_{2, \rho}^{1} \\
0 & 0 & -\sum_{\rho} x_{1, \rho}^{1}
\end{array}\right),\right. \\
& \times=2, \ldots, n\left(\begin{array}{ccc}
x_{1, \rho}^{1} & x_{2, \rho}^{1} & x_{3, \rho}^{1} \\
0 & -\sum_{\sigma \neq \rho} x_{1, \sigma}^{1}-x_{2,1}^{2} & -x_{2,1}^{1} \\
0 & -x_{1,1}^{2} & -\sum_{\beta \neq \rho} x_{1, \beta}^{1}
\end{array}\right) \mid \sum_{\alpha} x_{3, \alpha}^{1}=0
\end{aligned}
$$


Here the index ranges are $1 \leq \alpha, \beta \leq n, 2 \leq \rho, \sigma \leq n$. Note $\operatorname{dim} \mathfrak{g}_{p_{(\mathrm{iv})}}=3 n+1$.

\subsection{Second proof of dimensions in Theorem 1.2}

Throughout this section $X=\operatorname{Seg}\left(\mathbb{P} A_{1} \times \cdots \times \mathbb{P} A_{n}\right)$.

We show the assertion about the codimension of types (ii), (iii), (iv). Type (ii) is immediate as its closure is $J(X, \tau(X))$ which is easily seen to have the expected dimension via Terracini's lemma.

We will use the following lemma:

Lemma 5.2 Suppose $n \geq 2$ and $\operatorname{dim} A_{i} \geq 3$ for all $i \in\{1, \ldots, n\}$. Let $R$ be a degree 3 , zero-dimensional subscheme of $X=\operatorname{Seg}\left(\mathbb{P} A_{1} \times \cdots \times \mathbb{P} A_{n}\right)$. Suppose moreover $R$ is in general position, that is, it is not contained in any $\operatorname{Seg}\left(\mathbb{P} A_{1} \times \cdots \times \mathbb{P}^{1} \times \cdots \times \mathbb{P} A_{n}\right)$. Let $\langle R\rangle \simeq \mathbb{P}^{2} \subset \mathbb{P}\left(A_{1} \otimes \cdots \otimes A_{n}\right)$ denote the smallest linear space containing $R$. Then $X \cap\langle R\rangle=R$.

Proof Any such $R$ is isomorphic either to 3 distinct reduced points, or a double point and a reduced point, or one of the two kinds of triple points: $\operatorname{Spec} \mathbb{C}[x] / x^{3}$, or $\operatorname{Spec} \mathbb{C}[x, y] /\left\langle x^{2}, x y, y^{2}\right\rangle$.

If $n=2$, without loss of generality, we may suppose $\operatorname{dim} A_{1}=\operatorname{dim} A_{2}=3$. We can write down explicitly $\langle R\rangle \subset \mathbb{P}\left(A_{1} \otimes A_{2}\right)$ for each of the schemes as, respectively:

$$
\left(\begin{array}{lll}
s & & \\
& t & \\
& & u
\end{array}\right), \quad\left(\begin{array}{lll}
t & s & \\
s & & \\
& & u
\end{array}\right),\left(\begin{array}{lll}
u & t & s \\
t & s & \\
s & &
\end{array}\right),\left(\begin{array}{lll}
t & s & u \\
s & & \\
u & &
\end{array}\right) .
$$

The claim may be verified explicitly for each case, by calculating the scheme defined by $2 \times 2$ minors of each of the matrices.

If $n \geq 3$, let $B_{i}=A_{1} \otimes \cdots \otimes A_{i-1} \otimes A_{i+1} \otimes \cdots \otimes A_{n}$. Then $X=\bigcap_{i=1}^{n} \mathbb{P} A_{i} \times \mathbb{P} B_{i}$, and the claim easily follows from the $n=2$ statement.

Lemma 5.3 Suppose $n \geq 2$ and $\operatorname{dim} A_{i} \geq 3$ for all $i \in\{1, \ldots, n\}$. Let $X=$ $\operatorname{Seg}\left(\mathbb{P} A_{1} \times \cdots \times \mathbb{P} A_{n}\right)$ and let $\ell \subset X$ be a line spanned by $x, y \in X$. Let $v \in$ $\hat{T}_{x} X+\hat{T}_{y} X$ be general and consider $\mathbb{P}^{2}$ spanned by $\ell$ and $[v]$. Then $\mathbb{P}^{2} \cap X=\ell$.

Proof Let $x=a_{1}^{1} \otimes \cdots \otimes a_{1}^{n}, y=a_{2}^{1} \otimes a_{1}^{2} \otimes \cdots \otimes a_{1}^{n}$, and $v$ be as in (1.5). Let $B:=$ $A_{2} \otimes \cdots \otimes A_{n}$ and:

$$
\begin{aligned}
& b_{1}:=a_{1}^{2} \otimes \cdots \otimes a_{1}^{n}, \\
& b_{2}:=\sum_{i=2}^{n} a_{1}^{2} \otimes \cdots \otimes a_{1}^{i-1} \otimes a_{2}^{i} \otimes a_{1}^{i+1} \otimes \cdots \otimes a_{1}^{n}, \\
& b_{3}:=\sum_{i=2}^{n} a_{1}^{2} \otimes \cdots \otimes a_{1}^{i-1} \otimes a_{3}^{i} \otimes a_{1}^{i+1} \otimes \cdots \otimes a_{1}^{n} .
\end{aligned}
$$


Then $x=a_{1}^{1} \otimes b_{1}, y=a_{2}^{1} \otimes b_{1}$ and $v=a_{1}^{1} \otimes b_{3}+a_{2}^{1} \otimes b_{2}+a_{3}^{1} \otimes b_{1}$. Consider a linear combination $s v+t x+u y$. The intersection $\mathbb{P}^{2} \cap X$ is contained in the zero locus of the $2 \times 2$ minors of the following matrix:

$$
\left(\begin{array}{lll}
t & & s \\
u & s & \\
s & &
\end{array}\right),
$$

which can be identified with the line $s=0$ that is, the line spanned by $x$ and $y$.

Let $\operatorname{Osc}(X)$ be the closure of the set of points of type (iii). Let $[p] \in \operatorname{Osc}(X)$ be a general point. We claim such $p$ uniquely determines $[x]$ such that $p=x+x^{\prime}+x^{\prime \prime}$. Suppose without loss of generality $\operatorname{dim} A_{1}=3$. Write $p=p_{\text {(iii) }}$ of (1.4), and consider the underlying map $p_{\text {(iii) }}: A_{1}{ }^{*} \rightarrow A_{2} \otimes \cdots \otimes A_{n}$ :

$$
\begin{aligned}
p_{(\mathrm{iii})}\left(a_{1}^{1^{*}}\right)= & \sum_{2 \leq i<j}^{n} a_{1}^{2} \otimes \cdots \otimes a_{1}^{i-1} \otimes a_{2}^{i} \otimes a_{1}^{i+1} \otimes \cdots \otimes a_{1}^{j-1} \otimes a_{2}^{j} \otimes a_{1}^{j+1} \otimes \cdots \otimes a_{1}^{n} \\
& +\sum_{i=2}^{n} a_{1}^{2} \otimes \cdots \otimes a_{1}^{i-1} \otimes a_{3}^{i} \otimes a_{1}^{i+1} \otimes \cdots \otimes a_{1}^{n}, \\
p_{(\mathrm{iii})}\left(a_{2}^{1^{*}}\right)= & \sum_{j=2}^{n} a_{1}^{1} \otimes \cdots \otimes a_{1}^{j-1} \otimes a_{2}^{j} \otimes a_{1}^{j+1} \otimes \cdots \otimes a_{1}^{n}, \\
p_{(\mathrm{iii})}\left(a_{3}^{1^{*}}\right)= & a_{1}^{2} \otimes \cdots \otimes a_{1}^{n} .
\end{aligned}
$$

The projectivization of the image is a $\mathbb{P}^{2}$ containing a degree 3 scheme $R \subset$ $\operatorname{Seg}\left(\mathbb{P} A_{2} \times \cdots \times \mathbb{P} A_{n}\right)$ in general position, which is isomorphic to the triple point Spec $\mathbb{C}[x] / x^{3}$ supported at $\left[p\left(a_{3}^{1^{*}}\right)\right]$. By Lemma $5.2, R$ is determined by $\langle R\rangle=$ $\mathbb{P}\left(p\left(A_{1}^{*}\right)\right)$, so it is independent of the choice of normal form. Therefore $\left\langle a_{2}^{1^{*}}, a_{3}^{1^{*}}\right\rangle$, which is the linear span of the unique degree 2 subscheme of $R$, is determined by $p$, and so is $a_{1}^{1}$ (up to scale). Similarly, $a_{1}^{i}$ are determined by $p$ up to scale.

Thus we have a rational dominant map $\psi: \operatorname{Osc}(X) \rightarrow X, \psi(p):=\left[a_{1}^{1} \otimes \cdots \otimes\right.$ $\left.a_{1}^{n}\right]$. A general fiber over $o \in X$ is contained in the second osculating space $\mathbb{P}(\hat{o} \oplus$ $\left.T \oplus N_{2}\right)$, and its closure is equal to the closure of points of the form $\bar{o}+\tilde{\xi}^{\prime}+I I\left(\tilde{v}^{2}\right)$. Thus $\operatorname{dim} O \operatorname{Osc}(X)=3 \sum\left(\operatorname{dim} A_{i}-1\right)$.

Finally consider $\mathrm{Z}(X)$, the set of points of type (iv), which is closed by Lemma 3.4. Let $[p] \in \mathrm{Z}(X)$ be a general point of any of the irreducible components. We claim $p$ uniquely determines the line $\mathbb{P}\langle x, y\rangle$ such that $p=x+x^{\prime}+y+y^{\prime}$. Suppose without loss of generality $\operatorname{dim} A_{i}=3$ for all $i$. Possibly permuting the factors, write $p=p_{\text {(iv) }}$ of (1.5). First consider the underlying map $p_{(\mathrm{iv})}: A_{1}^{*}: \rightarrow A_{2} \otimes \cdots \otimes A_{n}$ :

$$
\begin{aligned}
& p_{\text {(iv) }}\left(a_{1}^{1^{*}}\right)=\sum_{i=2}^{n} a_{1}^{2} \otimes \cdots \otimes a_{1}^{i-1} \otimes a_{3}^{i} \otimes a_{1}^{i+1} \otimes \cdots \otimes a_{1}^{n}, \\
& p_{\text {(iv) }}\left(a_{2}^{1^{*}}\right)=\sum_{i=2}^{n} a_{1}^{2} \otimes \cdots \otimes a_{1}^{i-1} \otimes a_{2}^{i} \otimes a_{1}^{i+1} \otimes \cdots \otimes a_{1}^{n},
\end{aligned}
$$




$$
p_{\text {(iv) }}\left(a_{3}^{1^{*}}\right)=a_{1}^{2} \otimes \cdots \otimes a_{1}^{n}
$$

The projectivization of the image is a $\mathbb{P}^{2}$ containing a degree 3 scheme $R \subset$ $\operatorname{Seg}\left(\mathbb{P} A_{2} \times \cdots \times \mathbb{P} A_{n}\right)$ in general position, which is isomorphic to the triple point Spec $\mathbb{C}[x, y] /\left\langle x^{2}, x y, y^{2}\right\rangle$ point supported at $\left[p_{(\mathrm{iv})}\left(a_{3}^{1^{*}}\right)\right]$. By Lemma 5.2, $\left[p_{(\mathrm{iv})}\left(a_{3}^{1^{*}}\right)\right]$ is the unique reduced point in $\mathbb{P}\left(p_{(\text {iv })}\left(A_{1}^{*}\right)\right) \cap \operatorname{Seg}\left(\mathbb{P} A_{2} \times \cdots \times \mathbb{P} A_{n}\right)$, so independent of the choice of normal form. Therefore $\left\langle a_{1}^{1}, a_{2}^{1}\right\rangle \subset \mathbb{P} A_{1}$ is determined by $p_{\text {(iv) }}$.

Now consider $p_{(\text {iv })}: A_{n}^{*}: \rightarrow A_{1} \otimes \cdots \otimes A_{n-1}$ :

$$
\begin{aligned}
p_{\text {(iv) }}\left(a_{1}^{n *}\right)= & \sum_{i=1}^{n-1} a_{1}^{1} \otimes \cdots \otimes a_{1}^{i-1} \otimes a_{3}^{i} \otimes a_{1}^{i+1} \otimes \cdots \otimes a_{1}^{n-1} \\
& +\sum_{i=2}^{n-1} a_{2}^{1} \otimes a_{1}^{2} \otimes \cdots \otimes a_{1}^{i-1} \otimes a_{2}^{i} \otimes a_{1}^{i+1} \otimes \cdots \otimes a_{1}^{n-1}, \\
p_{\text {(iv) }}\left(a_{2}^{n *}\right)= & a_{2}^{1} \otimes a_{1}^{2} \otimes \cdots \otimes a_{1}^{n-1}, \\
p_{\text {(iv) }}\left(a_{3}^{n *}\right)= & a_{1}^{1} \otimes \cdots \otimes a_{1}^{n-1} .
\end{aligned}
$$

By Lemma 5.3 the projective line $\mathbb{P}\left\langle p_{(\mathrm{iv})}\left(a_{2}^{n *}\right), p_{(\mathrm{iv})}\left(a_{3}^{n *}\right)\right\rangle$ is determined by $p_{(\mathrm{iv})}$. Thus $a_{1}^{n}$ (and similarly $a_{1}^{i}$ for $i \in\{2, \ldots, n\}$ ) is determined (up to scale) by $p_{\text {(iv) }}$. Therefore, the line $\mathbb{P}\left(\left\langle a_{1}^{1}, a_{2}^{1}\right\rangle \otimes a_{1}^{2} \otimes \cdots \otimes a_{1}^{n}\right) \subset X$ is uniquely determined by $p_{\text {(iv) }}$.

The lines on $X$ are parameterized by $n$ irreducible varieties:

$$
L_{i}:=\mathbb{P} A_{1} \times \cdots \times \mathbb{P} A_{i-1} \times G\left(2, A_{i}\right) \times \mathbb{P} A_{i+1} \times \cdots \times \mathbb{P} A_{n} .
$$

By the argument above we have a rational dominant map $\chi: \mathrm{Z}(X) \rightarrow L_{1} \sqcup \cdots \sqcup L_{n}$. A general fiber over $\ell \in L_{i}$ is $\mathbb{P} \mathcal{T}^{\ell}$ in the notation of Lemma 3.3, the linear span of projective tangent spaces to $X$ at points of $\ell$. By (5.1) $\operatorname{dim} \mathcal{T}^{\ell}=2 \operatorname{dim} X+2-\operatorname{dim} A_{i}$, and the dimension of each irreducible component of $\mathrm{Z}(X)$ is equal to $3 \sum\left(\operatorname{dim} A_{i}-\right.$ $1)-2$.

\section{Orbits of tensors in $A \otimes B \otimes C$ of border rank at most 3}

Let $A \simeq \mathbb{C}^{\mathbf{a}}, B \simeq \mathbb{C}^{\mathbf{b}}, C \simeq \mathbb{C}^{\mathbf{c}}$. Let

$$
\begin{aligned}
& S u b_{\mathbf{a}^{\prime}, \mathbf{b}^{\prime}, \mathbf{c}^{\prime}}=S u b_{\mathbf{a}^{\prime}, \mathbf{b}^{\prime}, \mathbf{c}^{\prime}}(A \otimes B \otimes C) \\
& =\left\{T \in A \otimes B \otimes C \mid \exists \mathbb{C}^{\mathbf{a}^{\prime}} \subset A, \mathbb{C}^{\mathbf{b}^{\prime}} \subset B, \mathbb{C}^{\mathbf{c}^{\prime}} \subset C,\right. \\
& \text { such that } \left.T \in \mathbb{C}^{\mathbf{a}^{\prime}} \otimes \mathbb{C}^{\mathbf{b}^{\prime}} \otimes \mathbb{C}^{\mathbf{c}^{\prime}}\right\}
\end{aligned}
$$

This subspace variety admits a desingularization as follows. Let $\mathcal{E} \rightarrow G\left(\mathbf{a}^{\prime}, A\right) \times$ $G\left(\mathbf{b}^{\prime}, B\right) \times G\left(\mathbf{c}^{\prime}, C\right)$ be $\mathcal{E}=\mathcal{S}_{A} \otimes \mathcal{S}_{B} \otimes \mathcal{S}_{C}$, where $\mathcal{S}_{A} \rightarrow G\left(\mathbf{a}^{\prime}, A\right)$ is the tautological rank $\mathbf{a}^{\prime}$ subspace bundle and similarly for $B, C$. Then $\mathbb{P} \mathcal{E} \rightarrow S u b_{\mathbf{a}^{\prime}, \mathbf{b}^{\prime}, \mathbf{c}^{\prime}}(A \otimes B \otimes C)$ is a desingularization and using it one can see that $\operatorname{Sub}_{\mathbf{a}^{\prime}, \mathbf{b}^{\prime}, \mathbf{c}^{\prime}}(A \otimes B \otimes C)_{\text {sing }}=$ 
Table 1 Orbits of border rank 3 in $A \otimes B \otimes C$ that are not contained in a $S u b_{233}$, $S u b_{323}$, or $S u b_{332}$. Orbits 34-36 are identical up to permutations of $A, B, C$

\begin{tabular}{|c|c|c|c|c|c|c|}
\hline \# & Orbit closure & $\operatorname{dim}$ & Normal form & Slice & $\underline{\mathbf{R}}$ & $\mathbf{R}$ \\
\hline 34 & $\mathrm{Z}(X)_{A}$ & $3 \mathbf{a}+3 \mathbf{b}+3 \mathbf{c}-11$ & $\begin{array}{l}a_{1} \otimes\left(b_{1} \otimes c_{2}+b_{2} \otimes c_{1}\right)+a_{2} \otimes b_{1} \otimes c_{1} \\
+a_{3} \otimes\left(b_{3} \otimes c_{1}+b_{1} \otimes c_{3}\right)\end{array}$ & & 3 & 5 \\
\hline 35 & $\mathrm{Z}(X)_{B}$ & $3 \mathbf{a}+3 \mathbf{b}+3 \mathbf{c}-11$ & $\begin{array}{l}a_{1} \otimes\left(b_{1} \otimes c_{2}+b_{2} \otimes c_{1}+b_{3} \otimes c_{3}\right) \\
+a_{2} \otimes b_{1} \otimes c_{1}+a_{3} \otimes b_{3} \otimes c_{1}\end{array}$ & & 3 & 5 \\
\hline 36 & $\mathrm{Z}(X)_{C}$ & $3 \mathbf{a}+3 \mathbf{b}+3 \mathbf{c}-11$ & $\begin{array}{l}a_{1} \otimes\left(b_{1} \otimes c_{2}+b_{2} \otimes c_{1}+b_{3} \otimes c_{3}\right) \\
+a_{2} \otimes b_{1} \otimes c_{1}+a_{3} \otimes b_{1} \otimes c_{3}\end{array}$ & & 3 & 5 \\
\hline 37 & $\operatorname{Osc}(X)$ & $3 \mathbf{a}+3 \mathbf{b}+3 \mathbf{c}-9$ & $\begin{array}{l}a_{1} \otimes\left(b_{1} \otimes c_{3}+b_{2} \otimes c_{2}+b_{3} \otimes c_{1}\right) \\
+a_{2} \otimes\left(b_{1} \otimes c_{2}+b_{2} c_{1}\right)+a_{3} \otimes b_{1} \otimes c_{1}\end{array}$ & & 3 & 5 \\
\hline 38 & $J(X, \tau(X))$ & $3 \mathbf{a}+3 \mathbf{b}+3 \mathbf{c}-8$ & $\begin{array}{l}a_{1} \otimes\left(b_{1} \otimes c_{2}+b_{2} \otimes c_{1}\right) \\
+a_{2} \otimes b_{1} \otimes c_{1}+a_{3} \otimes b_{3} \otimes c_{3}\end{array}$ & & 3 & 4 \\
\hline 39 & $\sigma_{3}(X)$ & $3 \mathbf{a}+3 \mathbf{b}+3 \mathbf{c}-7$ & $a_{1} \otimes b_{1} \otimes c_{1}+a_{2} \otimes b_{2} \otimes c_{2}+a_{3} \otimes b_{3} \otimes c_{3}$ & & 3 & 3 \\
\hline
\end{tabular}

$S u b_{\mathbf{a}^{\prime}-1, \mathbf{b}^{\prime}, \mathbf{c}^{\prime}} \cup \operatorname{Sub} b_{\mathbf{a}^{\prime}, \mathbf{b}^{\prime}-1, \mathbf{c}^{\prime}} \cup S u b_{\mathbf{a}^{\prime}, \mathbf{b}^{\prime}, \mathbf{c}^{\prime}-1}$, whenever $\mathbf{a}^{\prime}<\mathbf{b c}$, and similarly for permuted statements. In [4, §6], normal forms for tensors in $S_{u} b_{233} \cup S_{u} b_{323} \cup S_{u b} b_{32}$ are given. There are 33 such.

We present the list of remaining orbits in $\sigma_{3}(\operatorname{Seg}(\mathbb{P} A \times \mathbb{P} B \times \mathbb{P} C))$ under the action of $G L(A) \times G L(B) \times G L(C)$.

Each orbit is uniquely determined by its closure, which is an algebraic variety listed in the second column of the table. The orbit itself is an open dense subset of this variety. The dimension of the algebraic variety is in the third column. The fourth column is the normal form of the underlying tensor, the distinct variables are assumed to be linearly independent. The normal form is also given as a slice. The border rank and rank are given in the next columns.

$\mathrm{Z}(X)_{A}, \mathrm{Z}(X)_{B}, \mathrm{Z}(X)_{C}$ denote the three components of $\mathrm{Z}(X)$, the set points of type (iv) in Theorem 1.2. Osc( $X)$ denotes the closure of the set points of type (iii), while $J(X, \tau(X))$ denotes the closure of the set points of type (ii).

The ranks of cases 34-37 in Table 1 are calculated in Sect. 6.1. The rank of case 39 is obvious, while the rank of case 38 is at most 4, due to the normal form expression. If it were 3 , then a general point of type (ii), would be expressible as a point of type (i), a contradiction with Theorem 1.2.

\subsection{Proof of Theorem 1.8}

The rank of a linear subspace $U \subset \mathbb{C}^{k} \otimes \mathbb{C}^{l}$ is the smallest $r$ such that $U$ is contained in a linear space of dimension $r$ spanned by rank-one elements. The rank of a tensor $T \in A \otimes B \otimes C$ equals the rank of the linear subspace $T\left(A^{*}\right) \subset B \otimes C$ (see, e.g., [15, Thm. 3.1.1.1]). 
Proposition 6.1 The ranks of the spaces parameterized by

$$
\left(\begin{array}{lll}
u & t & s \\
t & s & 0 \\
s & 0 & 0
\end{array}\right)
$$

and by

$$
\left(\begin{array}{lll}
t & s & u \\
s & 0 & 0 \\
u & 0 & 0
\end{array}\right)
$$

are both 5 .

Proof We first show the rank is at most 5: in the second case, it is immediate. In the first case the rank of

$$
\left(\begin{array}{lll}
0 & t & s \\
t & s & 0 \\
s & 0 & 0
\end{array}\right)
$$

is 4 (see $[4, \S 6])$, and the rank of

$$
\left(\begin{array}{lll}
u & 0 & 0 \\
0 & 0 & 0 \\
0 & 0 & 0
\end{array}\right)
$$

is one.

To see the ranks are at least five, were it four in the first case, we would be able to find a $3 \times 3$ matrix

$$
T=\left(\begin{array}{lll}
f_{1} g_{1} & f_{1} g_{2} & f_{1} g_{3} \\
f_{2} g_{1} & f_{2} g_{2} & f_{2} g_{3} \\
f_{3} g_{1} & f_{3} g_{2} & f_{3} g_{3}
\end{array}\right)
$$

of rank 1, such that the 4-plane spanned by:

$$
T_{1}:=\left(\begin{array}{ccc}
0 & 0 & 1 \\
0 & 1 & 0 \\
1 & 0 & 0
\end{array}\right), \quad T_{2}:=\left(\begin{array}{ccc}
0 & 1 & 0 \\
1 & 0 & 0 \\
0 & 0 & 0
\end{array}\right), \quad T_{3}:=\left(\begin{array}{ccc}
1 & 0 & 0 \\
0 & 0 & 0 \\
0 & 0 & 0
\end{array}\right), \quad T
$$

is spanned by matrices of rank 1 . In particular, $T_{1}$ would be in the span of $T_{2}, T_{3}, T$ and another matrix of rank 1 . Thus we would be able to find constants $\beta, \gamma, f_{1}, f_{2}, f_{3}, g_{1}, g_{2}, g_{3}$, such that the rank of

$$
\left(\begin{array}{lll}
\gamma & \beta & 1 \\
\beta & 1 & 0 \\
1 & 0 & 0
\end{array}\right)+\left(\begin{array}{lll}
f_{1} g_{1} & f_{1} g_{2} & f_{1} g_{3} \\
f_{2} g_{1} & f_{2} g_{2} & f_{2} g_{3} \\
f_{3} g_{1} & f_{3} g_{2} & f_{3} g_{3}
\end{array}\right)
$$


is one. There are two cases: if $g_{3} \neq 0$, then we can subtract $\frac{g_{1}}{g_{3}}$ times the third column from the first, and $\frac{g_{2}}{g_{3}}$ times the third column from the second to obtain

$$
\left(\begin{array}{ccc}
* & * & 1+f_{1} g_{3} \\
* & 1 & f_{2} g_{3} \\
1 & 0 & f_{2} g_{3}
\end{array}\right)
$$

which has rank at least two. If $g_{3}=0$ the matrix already visibly has rank at least two. Thus it is impossible to find such constants $\beta, \gamma, f_{i}, g_{i}$ and the rank in question is necessarily at least 5 .

The second case is more delicate. Write all $2 \times 2$ minors of

$$
\left(\begin{array}{ccc}
t & s & u \\
s & 0 & 0 \\
u & 0 & 0
\end{array}\right)+x\left(\begin{array}{lll}
f_{1} g_{1} & f_{1} g_{2} & f_{1} g_{3} \\
f_{2} g_{1} & f_{2} g_{2} & f_{2} g_{3} \\
f_{3} g_{1} & f_{3} g_{2} & f_{3} g_{3}
\end{array}\right)
$$

and consider $f_{i}$ and $g_{j}$ as parameters of degree 0 , and remaining variables $\alpha_{1}, \alpha_{2}, \alpha_{3}, x$ of degree 1 . We claim $\left(s f_{3}-u f_{2}\right)^{2}$ and $\left(s g_{3}-u g_{2}\right)^{2}$ are in the ideal $\mathcal{I}$ generated by minors. This can be verified by patient calculation, or using a computer algebra system, such as Magma [2]. Thus $f_{2}=f_{3}=g_{2}=g_{3}=0$, for otherwise we have a degree 1 equation in the radical ideal $\sqrt{I}$, and then the rank-one matrices do not span the four-dimensional linear space. But in such a case $u^{2}$ and $s^{2}$ are among the minors, giving $u$ and $s$ as linear equations in $\sqrt{I}$, a contradiction.

\subsection{Singularities}

In this subsection we prove Theorems 1.3 and 1.4. The strategy is uniform to most cases: using the parameterization $\mathcal{E} \rightarrow S u b_{i, j, k}$ as in the beginning paragraph of Sect. 6, which is biregular away from the locus $S u b_{i-1, j, k} \cup \operatorname{Sub} b_{i, j-1, k} \cup S u b_{i, j, k-1}$, we reduce statements to properties of secant varieties of low dimensional Segre products.

Proof of Theorem 1.3 First note that $\sigma_{2}(\operatorname{Seg}(\mathbb{P} A \times \mathbb{P} B \times \mathbb{P} C))=\operatorname{Sub} b_{2,2,2}$. In particular, any point of $\sigma_{2}\left(\operatorname{Seg}\left(\mathbb{P}^{1} \times \mathbb{P}^{1} \times \mathbb{P}^{1}\right)\right)=\mathbb{P}^{7}$ is a smooth point. Now just observe that $\left[a_{1} \otimes b_{1} \otimes c_{2}+a_{1} \otimes b_{2} \otimes c_{1}+a_{2} \otimes b_{1} \otimes c_{1}\right]$ is a smooth point of $S u b_{2,2,2}$, because it is not contained in $S u b_{2,2,1} \cup S u b_{2,1,2} \cup S u b_{1,2,2}$.

Similarly, in Theorem 1.4 if $\operatorname{dim} A=2$, then $\sigma_{3}(\operatorname{Seg}(\mathbb{P} A \times \mathbb{P} B \times \mathbb{P} C))=\operatorname{Sub} b_{2,3,3}$. A general point of each type (i)-(iv) is not contained in any of the smaller subspace varieties, so the same $\operatorname{argument}$ works. So we will assume $\operatorname{dim} A, \operatorname{dim} B, \operatorname{dim} C \geq 3$.

Lemma 6.2 Suppose $\operatorname{dim} A=\operatorname{dim} B=\operatorname{dim} C=3$. Then a general point of each component of points of type (iv) is a smooth point of $\sigma_{3}(\operatorname{Seg}(\mathbb{P} A \times \mathbb{P} B \times \mathbb{P} C))$.

Proof The only defining equations of $\sigma_{3}\left(\operatorname{Seg}\left(\mathbb{P}^{2} \times \mathbb{P}^{2} \times \mathbb{P}^{2}\right)\right)$ are the 27 (degree four) Strassen equations. If we write $T=a_{1} \otimes X+a_{2} \otimes Y+a_{3} \otimes Z$, then 9 of the equations 
are the entries of the $3 \times 3$ matrix

$$
P(T)_{t}^{s}=\sum_{j, k}(-1)^{j+k}\left(\operatorname{det} X_{\hat{k}}^{\hat{j}}\right)\left(Y_{t}^{j} Z_{k}^{s}-Y_{k}^{s} Z_{t}^{j}\right)
$$

where $X_{\hat{k}}^{\hat{j}}$ is $X$ with its $j$-th row and $k$-th column removed. The remaining equations come from permuting the roles of $X, Y, Z$, see, e.g. [14]. Take $T=a_{1} \otimes\left(b_{1} \otimes c_{2}+\right.$ $\left.b_{2} \otimes c_{1}+b_{3} \otimes c_{3}\right)+a_{2} \otimes b_{1} \otimes c_{1}+a_{3} \otimes b_{3} \otimes c_{1}$ as in Table 1 row 35. Writing $T=a_{1} \otimes X+a_{2} \otimes Y+a_{3} \otimes Z$, we have

$$
X=\left(\begin{array}{lll}
0 & 1 & 0 \\
1 & 0 & 0 \\
0 & 0 & 1
\end{array}\right), \quad Y=\left(\begin{array}{lll}
1 & 0 & 0 \\
0 & 0 & 0 \\
0 & 0 & 0
\end{array}\right), \quad Z=\left(\begin{array}{lll}
0 & 0 & 0 \\
0 & 0 & 0 \\
1 & 0 & 0
\end{array}\right)
$$

Then

$$
d P_{T}=\left(\begin{array}{ccc}
-d x_{2,3}+d y_{1,3}-d z_{1,2}+d z_{2,1} & -d z_{2,2} & d z_{2,3} \\
d y_{2,3}-d z_{2,2} & 0 & 0 \\
d x_{2,2}-d y_{2,1}+d y_{3,3}-d z_{3,2} & -d y_{2,2} & -d y_{2,3}
\end{array}\right)
$$

which indeed has six linearly independent differentials.

To argue for the other components, i.e., when $T$ is of the form 34 or 36 in Table 1, one can permute the factors $A, B$, and $C$.

Proof of Theorem 1.4 Assume $\operatorname{dim} A, \operatorname{dim} B, \operatorname{dim} C \geq 3$. Since the map $\mathbb{P}(\mathcal{E}) \rightarrow$ $\mathrm{Sub}_{3,3,3}$ is an isomorphism near a general point of type (iv), the Lemma implies that such a point is a smooth point of $\sigma_{3}(\operatorname{Seg}(\mathbb{P} A \times \mathbb{P} B \times \mathbb{P} C)$ ) for any $A, B, C$ (each of dimension at least 3). But orbits 34-36 from Table 1 are in the closure of orbits 37 and 38. So $\sigma_{3}(\operatorname{Seg}(\mathbb{P} A \times \mathbb{P} B \times \mathbb{P} C))$ is non-singular at a general point of each type (ii)-(iv).

The final thing to prove is that $\sigma_{3}(\operatorname{Seg}(\mathbb{P} A \times \mathbb{P} B \times \mathbb{P} C))$ is non-singular at a general point of $S_{43} b_{23}$. Let $p$ be such a point. Since $\sigma_{3}(\operatorname{Seg}(\mathbb{P} A \times \mathbb{P} B \times \mathbb{P} C)) \subset S u b_{333}$, we may assume $\operatorname{dim} A=\operatorname{dim} B=\operatorname{dim} C=3$. First note that $S u b_{233}$ is not contained in $J(X, \tau(X))$, as they are both irreducible, have the same dimension and $J(X, \tau(X)) \not \subset$ $S_{u b} 233$. So $p$ is not in $J(X, \tau(X))$. By Theorem 1.2, this implies that there exists an open neighborhood $U \subset \sigma_{3}(\mathbb{P} A \times \mathbb{P} B \times \mathbb{P} C)$ of $p$, such that in this neighborhood all points are of type (i).

Consider the dominant rational map

$$
\phi:(A \times B \times C)^{\times 3} \rightarrow \hat{\sigma}_{3}(\operatorname{Seg}(\mathbb{P} A \times \mathbb{P} B \times \mathbb{P} C))
$$

$\left(a_{1}, b_{1}, c_{1}\right),\left(a_{2}, b_{2}, c_{2}\right),\left(a_{3}, b_{3}, c_{3}\right) \mapsto a_{1} \otimes b_{1} \otimes c_{1}+a_{2} \otimes b_{2} \otimes c_{2}+a_{3} \otimes b_{3} \otimes c_{3}$

Let $W:=\phi^{-1}(U)$. Then $\left.\phi\right|_{W}: W \rightarrow U$ is a regular surjective map. The aim is to calculate the tangent map at any point in $\phi^{-1}(p)$. We commence with identifying $\phi^{-1}(p)$. Since $R_{X}(p)=3$, any point in $\phi^{-1}(p)$ will be contained in a fixed $\left(A^{\prime} \times\right.$ $\left.B^{\prime} \times C^{\prime}\right)^{\times 3}$ with $\operatorname{dim} A^{\prime}=2, \operatorname{dim} B^{\prime}=\operatorname{dim} C^{\prime}=3$ by [4, Cor. 2.2].

Write $p=\left[a_{1} \otimes b_{1} \otimes c_{1}+\left(a_{1}+a_{2}\right) \otimes b_{2} \otimes c_{2}+a_{2} \otimes b_{3} \otimes c_{3}\right]$ (see [4, §6]). We claim that this normal form is unique up to trivialities such as 7-dimensions worth 
Table 2 Singularities of $\sigma_{3}(\operatorname{Seg}(\mathbb{P} A \times \mathbb{P} B \times \mathbb{P} C))$. In the first column we list the tensor space, assuming $4 \leq \mathbf{a} \leq \mathbf{b} \leq \mathbf{c}$. In the second column we write the dimension of the secant variety. In the third column we present the upper bound on the dimension of the singular locus of the secant variety, which follows from our results in this section

\begin{tabular}{lll}
\hline$A \otimes B \otimes C$ & $\operatorname{dim} \sigma_{3}$ & $\operatorname{dim} \operatorname{Sin} \leq \leq$ \\
\hline $\mathbb{C}^{2} \otimes \mathbb{C}^{2} \otimes \mathbb{C}^{2}$ & 7 & -1 \\
$\mathbb{C}^{2} \otimes \mathbb{C}^{2} \otimes \mathbb{C}^{3}$ & 11 & -1 \\
$\mathbb{C}^{2} \otimes \mathbb{C}^{2} \otimes \mathbb{C}^{\mathbf{c}}$ & $3 \mathbf{c}+2$ & $2 \mathbf{c}+3$ \\
$\mathbb{C}^{2} \otimes \mathbb{C}^{3} \otimes \mathbb{C}^{3}$ & 17 & -1 \\
$\mathbb{C}^{2} \otimes \mathbb{C}^{3} \otimes \mathbb{C}^{\mathbf{c}}$ & $3 \mathbf{c}+8$ & $3 \mathbf{c}+4$ \\
$\mathbb{C}^{2} \otimes \mathbb{C}^{\mathbf{b}} \otimes \mathbb{C}^{\mathbf{c}}$ & $3 \mathbf{b}+3 \mathbf{c}-1$ & $\max \{3 \mathbf{b}+3 \mathbf{c}-9,2 \mathbf{b}+3 \mathbf{c}-2\}$ \\
$\mathbb{C}^{3} \otimes \mathbb{C}^{3} \otimes \mathbb{C}^{3}$ & 20 & 18 \\
$\mathbb{C}^{3} \otimes \mathbb{C}^{3} \otimes \mathbb{C}^{\mathbf{c}}$ & $3 \mathbf{c}+11$ & $3 \mathbf{c}+9$ \\
$\mathbb{C}^{3} \otimes \mathbb{C}^{\mathbf{b}} \otimes \mathbb{C}^{\mathbf{c}}$ & $3 \mathbf{b}+3 \mathbf{c}+2$ & $3 \mathbf{b}+3 \mathbf{c}$ \\
$\mathbb{C}^{\mathbf{a}} \otimes \mathbb{C}^{\mathbf{b}} \otimes \mathbb{C}^{\mathbf{c}}$ & $3 \mathbf{a}+3 \mathbf{b}+3 \mathbf{c}-7$ & $2 \mathbf{a}+3 \mathbf{b}+3 \mathbf{c}-6$ \\
\hline
\end{tabular}

of rescalings, and permutations of summands. By writing $p:\left(A^{\prime}\right)^{*} \rightarrow B^{\prime} \otimes C^{\prime}$, we

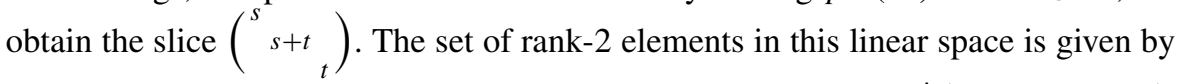
the determinant of the matrix. This set consists of three lines in $\left(A^{\prime}\right)^{*}$ spanned by $a_{1}^{*}$, $a_{1}^{*}-a_{2}^{*}$, and $a_{2}^{*}$. Thus the triple $a_{1},\left(a_{1}+a_{2}\right), a_{2}$ is (up to order and scale) determined by $p$. In a similar way we consider the other slices, and $2 \times 2$ minors of the resulting matrices, to conclude that triples $b_{1}, b_{2}, b_{3}$ and $c_{1}, c_{2}, c_{3}$ are determined by $p$, up to order and scale. It is easy to see, that any meaningfully different choice of orders, or scaling will give a different tensor, so the preimage of $p$ consists of 6 components, each of dimension 7 , isomorphic to $\left(\mathbb{C}^{*}\right)^{7}$.

Next, we calculate the image of tangent map of $\phi$ at any $q \in \phi^{-1}(p)$, say $q=$ $\left[\left(a_{1}, b_{1}, c_{1}\right),\left(a_{1}+a_{2}, b_{2}, c_{2}\right),\left(a_{2}, b_{3}, c_{3}\right)\right]$. This image is spanned by the following tensors, all considered modulo $p$, as we look at a subspace of $T_{p} \mathbb{P}(A \otimes B \otimes C) \simeq$ $(A \otimes B \otimes C) / p:$

$$
\begin{array}{lrll}
a_{i} \otimes b_{1} \otimes c_{1} & a_{i} \otimes b_{2} \otimes c_{2} & a_{i} \otimes b_{3} \otimes c_{3} & \text { for any } i \in\{1, \ldots, \operatorname{dim} A\}, \\
a_{1} \otimes b_{j} \otimes c_{1} & \left(a_{1}+a_{2}\right) \otimes b_{j} \otimes c_{2} & a_{2} \otimes b_{j} \otimes c_{3} & \text { for any } j \in\{1, \ldots, \operatorname{dim} B\}, \\
a_{1} \otimes b_{1} \otimes c_{k} & \left(a_{1}+a_{2}\right) \otimes b_{2} \otimes c_{k} & a_{2} \otimes b_{3} \otimes c_{k} & \text { for any } k \in\{1, \ldots, \operatorname{dim} C\} .
\end{array}
$$

This space is independent of the choice of the order or scalings in $q$. Also the linear space above has dimension

$$
3(\operatorname{dim} A+\operatorname{dim} B+\operatorname{dim} C)-7=\operatorname{dim} \sigma_{3}(\mathbb{P} A \times \mathbb{P} B \times \mathbb{P} C),
$$

because there are 3(dim $A+\operatorname{dim} B+\operatorname{dim} C)$ tensors listed above, and each $a_{1} \otimes b_{1} \otimes$ $c_{1},\left(a_{1}+a_{2}\right) \otimes b_{2} \otimes c_{2}, a_{2} \otimes b_{3} \otimes c_{3}$ is listed three times and $p$ is a sum of those three tensors. One can check there are no other linear dependencies.

Thus, $\phi: W \rightarrow \mathbb{P}(A \otimes B \otimes C)$ is a map with constant rank on an open subset containing $\phi^{-1}(p)$. Therefore the image is non-singular at $p$, as claimed. 
We summarize our results in Table 2 . In particular, it follows that $\sigma_{3}(\operatorname{Seg}(\mathbb{P} A \times$ $\mathbb{P} B \times \mathbb{P}())$ is always non-singular in codimension 1 , that is, codimension of the singular locus is at least 2. Moreover, it is of codimension 2 if and only if, one of the factors is $\mathbb{C}^{3}$, and the others have dimension at least 3 .

Acknowledgements J. Buczyński supported by Marie Curie Outgoing Fellowship "Contact Manifolds," Landsberg supported by NSF Grant DMS-1006353.

We thank M. Mohlenkamp for pointing out an error in an earlier version of this article, related to the rank of $y+y^{\prime}+y^{\prime \prime}$ in Theorem 1.8. This paper grew out of questions raised at the 2008 AIM workshop Geometry and Representation Theory of Tensors for Computer Science, Statistics and Other Areas, and the authors thank AIM and the conference participants for inspiration. The mathematics in this paper was finally completed while the authors were guests at the Mittag-Leffler Institute in Spring 2011 and we gratefully thank the institute for providing a wonderful environment for doing mathematics. We truly appreciate the help of the referee, his careful proofreading of the article, and his many thoughtful comments.

Open Access This article is distributed under the terms of the Creative Commons Attribution License which permits any use, distribution, and reproduction in any medium, provided the original author(s) and the source are credited.

\section{References}

1. Boralevi, A., Buczyński, J.: Secants of Lagrangian Grassmannians. Ann. Mat. Pura Appl. 1-15 (2010). doi:10.1007/s10231-010-0171-0

2. Bosma, W., Cannon, J., Playoust, C.: The Magma algebra system. I. The user language. J. Symb. Comput. 24(3-4), 235-265 (1997). Computational algebra and number theory (London, 1993). Available for use on-line at http://magma.maths.usyd.edu.au/calc/. MR 1484478

3. Buczyński, J., Ginensky, A., Landsberg, J.M.: Determinantal equations for secant varieties and the Eisenbud-Koh-Stillman conjecture. J. Lond. Math. Soc. (2) 88(1), 1-24 (2013). 14M12 (14N05)

4. Buczyński, J., Landsberg, J.M.: Ranks of tensors and a generalization of secant varieties. Linear Algebra Appl. 438(2), 668-689 (2013). 15A69 (14N05 15A21)

5. Cohen, A.M., Cooperstein, B.N.: Lie incidence systems from projective varieties. Proc. Am. Math. Soc. 126(7), 2095-2102 (1998). MR 1443819 (98h:14060)

6. Comon, P.: Tensor decompositions, state of the art and applications. In: McWhirter, J.G., Proudler, I.K. (eds.) Mathematics in Signal Processing V, pp. 1-24. Clarendon Press, Oxford (2002). arXiv:0905.0454v1

7. Fulton, W., Harris, J.: Representation Theory. Graduate Texts in Mathematics, vol. 129. Springer, New York (1991). A first course, Readings in Mathematics, MR 1153249 (93a:20069)

8. Ivey, T.A., Landsberg, J.M.: Cartan for Beginners: Differential Geometry via Moving Frames and Exterior Differential Systems. Graduate Studies in Mathematics, vol. 61. American Mathematical Society, Providence (2003). MR 2003610

9. Kolda, T.G., Bader, B.W.: Tensor decompositions and applications. SIAM Rev. 51(3), 455-500 (2009). MR 2535056

10. Kruskal, J.B.: Rank, decomposition, and uniqueness for 3-way and $N$-way arrays. In: Multiway Data Analysis (Rome, 1988), pp. 7-18. North-Holland, Amsterdam (1989). MR 1088949

11. Landsberg, J.M., Manivel, L.: On the projective geometry of rational homogeneous varieties. Comment. Math. Helv. 78(1), 65-100 (2003). MR 2004a:14050

12. Landsberg, J.M., Teitler, Z.: On the ranks and border ranks of symmetric tensors. Found. Comput. Math. 10(3), 339-366 (2010). MR 2628829 (2011d:14095)

13. Landsberg, J.M., Weyman, J.: On tangential varieties of rational homogeneous varieties. J. Lond. Math. Soc. (2) 76(2), 513-530 (2007). MR 2363430

14. Landsberg, J.M., Weyman, J.: On the ideals and singularities of secant varieties of Segre varieties. Bull. Lond. Math. Soc. 39(4), 685-697 (2007). MR 2346950

15. Landsberg, J.M.: The Geometry of Tensors: Applications to Complexity, Statistics and Engineering. Graduate Studies in Mathematics. AMS, Providence (2011)

16. Schönhage, A.: Partial and total matrix multiplication. SIAM J. Comput. 10(3), 434-455 (1981). MR 623057 (82h:68070) 